\title{
NASA THIXESSS24
}

THE SOURCE SPECTRA AND COMPOSITION OF COSMIC RAYS IMPLIED BY AN ANALYSIS OF INTERSTELLAR AND INTERPLANETARY TRAVEL

\section{GPO PRICE \$ \\ CFSTI PRICE(S) \$}

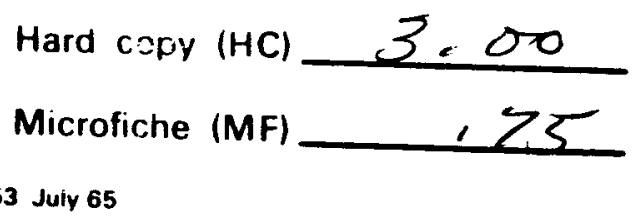

APRIL 1966 GREENBELT, MD.
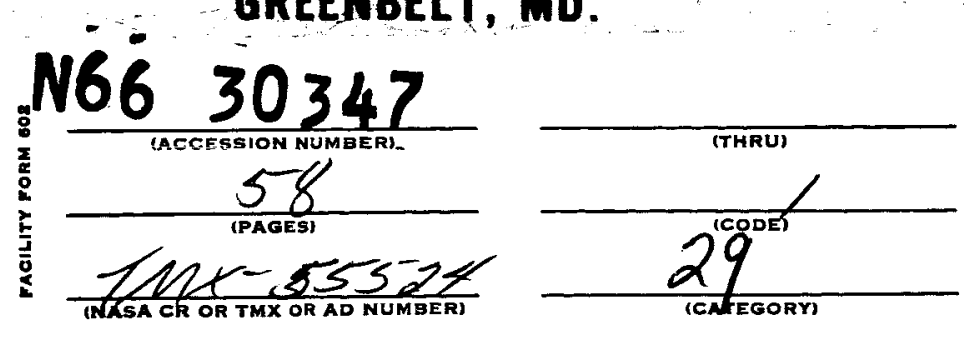
$\mathrm{X}-611-66-164$

THE SOURCE SPECTRA AND COMPOSITION

OF COSMIC RAYS

IMPLIED BY AN ANALYSIS

OF INTERSTELLAR AND INTERPLANETARY TRAVEL

by

C. E. Fichtel and D. V. Reames

Goddard Space Flight Center

Greenbelt, Maryland 
The Source Spectra and Composition

Of Cosmic Rays

Implied by an Analysis

of Interstellar and Interplanetary Travel

C. E. Fichtel and D. V. Reames

Goddard Space Flight Center, Greenbelt, Maryland

\begin{abstract}
The implications of a simple set of assumptions related to galactic cosmic rays are examined and compared to the existing data. These assumptions are that (a) the multiply charged cosmic ray nuclei all have the same spectral shape at the source, (b) the relative abundances of $\mathrm{He}^{3}$ and light nuclei $(3 \leq \mathrm{Z} \leq 5)$ are negligible at the source, and (c) the average amount of interstellar material traversed is independent of particle energy. The results show that within the present uncertainties of the experimental data and of the interaction cross sections, the data agree with the predictions without additional assumptions if the differential source spectra are relatively flat at low energies and the average interstellar mean free path is $2.8 \pm 0.4 \mathrm{~g} / \mathrm{cm}^{2}$. In particular, the nearly constant relative abundances of the helium, medium $(6 \leq z \leq 9)$, and $(z \geq 10)$ nuclei with energyinucleon can be explained, and the observed variation of the light to medium nuclei flux ratio with energy nucleon can be brought into fair agreement with predictions. The calculations also imply that, if the analysis does represent the true situation, then
\end{abstract}


there is little or no solar modulation of the cosmic rays near solar minimum, and protons and helium nuclei have different source spectra.

\section{Introduction}

The cosmic ray energy spectra which are observed at the earth represent the source spectra after they have passed through interstellar matter and have been modulated within the solar system. Whereas presumably the solar systen modulation is primarily the result of electromagnetic fields, interstellar space is believed to contain enough material along the path uf the particle to change appreciably the particle energy as well as the intensity of the radiation. In the latter case it is normally assumed that the intensity in an energy interval is changed significantly only by fragmentation in interactions and by ionization loss, and not by accelerntion nor by the complicated time-dependent magnetic effects which probably cause the intensity variation in the solar system.

If the acceleration in interstellar space is negligible, it is possible to calculate the energy dependence of the relative abundances of various particle groups outside the solar system, assuming various source spectra, provided the cross sections in interstellar space and the amount of materiai traversed are sufficiently well known. The exact nature of the solar modulation is not yet known, but the general belief is that it probably deiends only on the velocity and charge-to-mass ratio of the particle. Therefore, although nuclei of the same cherge-to-mass ratio but different charges will lose energy at different rates in interstellar space, the fluxes of these 
particles will be modulated in the same way, thereby permitting the separation of modulation effects from interstellar energy loss and fragmentation effects.

Enough information is now accumulating on several different components of the cosmic radiation to permit a meaningful study of their source spectra and history of the cosmic radiation can be obtained. Therefore, in this paper we have determined the expected relative abundances of the multiply charged nuclei as a function of energy nucleon for various specific assumptions about the source spectra and interstellar mean free path, and have also considered the effects of solar modulation. Throughout, the analysis has been made as quantitative as seems reasonable within the limited knowledge of some of the parameters.

\section{General Procedure}

The first purpose of this article is to determine whether a particular simple set of assumptions leads to predictions which are consistent with the galactic cosmic ray data. These assumptions are:

(a) The source energy/nucleon spectra of all multiply charged nuclei have the same shape, at least above $100 \mathrm{MeV} /$ nucleon. (Note that, since all of the multiply charged nuclei that will be of interest at the source have nearly the same charge to mass ratio, this effectively permits the spectra to be both velocity and rigidity dependent.)

(b) The relative abundance of $\mathrm{He}^{3}$ and light nuclei $(3 \leq \mathrm{Z} \leq 5)$ at the source are negligible compared to $\mathrm{He}^{4}$ and medium nuclei respectively. 
(c) The average interstellar mean free path is independent of the energy/nucleon of the particle.

After a thorough investigation of this simple approach and its limitations, we consider alternate approaches and models (usually involving more adjustable parameters) suggested in the literature.

Before out lining the general procedure, we shall briefly discuss the assumptions. The similarity in source spectral shave, at least for particles of the same charge to mass ratio, is suggested by the predictions of the basic acceleration mechanisms, such as the simple Fermi ${ }^{1,2}$ theory, the principle of equipartition of energy as discussed by syrovatsky ${ }^{3}$, and the theory of the shock acceleration of the outer layer of a supernovae discussed by Colgate and Johnson ${ }^{4}$ and Colgate 5 . Some of the more complicated variations of the theories can explain differences in the energy stectra of different nuclei, especially those with different charge-to-mass ratio. Further support to the choice of spectra of the same shape for particles of the same charge-to-mass ratio is obtained from the study of solor particles. It should be noted that source spectra at very low energies $\left(<10^{2} \mathrm{MeV} /\right.$ nucleon) may differ in general, but they are not germane to the present discussion because, as will be discussed later, they could not be detected.

With respect to the composition, the only important assumption that we shall make is that $1 \mathrm{ight}$ nucle $\mathrm{i}$ and $\mathrm{He}^{3}$ are absent in the source. For the light nuclei, this assumption is based on the foct that 1 ight nuclei are very rare in the universe (about $10^{-5}$ of the abundance of medium 
nuclei) ${ }^{8}$ because they are unstable at the high temperatures of most stars. The justification for assuming there is virtually no $\mathrm{He}^{3}$ at the source is similar.

Finally, the assumption regarding the average mean free path being independent of energy is based on simplicity and the fact that in a simple steady state diffusion process, the path length would not be expected to vary with velocity. A rigidity dependent average path length is of course possible if, for example, high rigidity particles escape more easily. This alternate possibility will be one of the ones considered in section $\mathrm{V}$.

In proceeding with the calculations, the problem will be divided into three parts or physical regions: the "source" which suppiies the cosmic radiation, the interstellar medium containing primarily hydrogen which can cause fragmentation and energy loss of the cosmic-ray particles, and finally the Solar System which influences the cosmic-ray fluxes via its electromagnetic fields.

With regard to the source, it will be initially assumed that the source spectra have the properties stated in assumptions (a) and (b), and the remaining characteristics, such as the possible shapes for the energy spectr: and the relative abundances of the other nuclear species, will be determined from an examination of the experimental data in the light of the calculation to follow. Therefore, the first consideration will be the travel of the cosmic rays through interstellar space, which will be discussed in section III. As was stated in the introduction, the model being discussed here does not allow the interstellar magnetic fields to accelerate the 
particles, but does permit diffusion. The interstellar path length to be determined is defined as the average number of $\mathrm{g} / \mathrm{cm}^{2}$ of material traversed by the particles. The interstellar extrapolation includes both the effects of energy luss by ionization and of fragmentation which is dependent on the energy of the cosmic rays.

In section IV, the effect of solar moduiation is considered. Various possible types of modulation are considered both from the standpoint of the cxtent of the sular modulation and its possible effect on particle ratios as a function of energy. It will be seen that the relative abundances of the multiply charged nuclei are probably not seriously affected by modulation, owing to the similar charge-to-mass ratios of the constitvents of interest. On the other hand, particles with the same $7^{2} / M$ value ( $Z$ and $M$ are the charge and mass of the particles in units of the proton charge and mass), such as protons and $\mathrm{He}^{4}$, will have the same rate of energy loss per nucleon for a given velocity in interstellar space, but will be inodulated by different amounts. The alternate approach of demodulating the observed spectra and then extrapolating to the sources requires a quantitative knowledge of the modulation.

In $\operatorname{section} V$, the experimental data are compared to the predictions trom the calculations. It will be seen that the predictions essentially agree with the experimental data within the uncertainty of the measurements fur a specific set of relative abundances and a limited range of energy spectra. Other possible theoretical explanations of the data are also considered. 


\section{Interstellar Extrapolation}

The material in interstellar space affects the cosmic-ray particles by causing energy loss and fragmentation. In order to take both of these phenomena into account, it is necessary to begin with the appropriate transport equation. $\operatorname{Ray}^{9}$ has shown that the fundamental equation can be written in the form

$$
\frac{d}{d x}\left[w_{i}(E) j_{i}(E, x)\right]=w_{i}(E) H_{i}(E, x),
$$

where $x$ is the position aiong the particle path, $w_{i}=(d E / d x)_{i}, i$ refers to the particular nuclear species, $j_{i}$ is the differential directional intensity per unit energy/nucleon, $E$, for particles of type $i$, and $H_{i} i s$ the number of particles added or subtracted per unit volune, time, solid angle, and energy nucleon.

In the case under consideration $H_{i}$ consists of two parts, the source term $S_{i}$ and the loss term due to collisions. Hence,

$$
H_{i}(E, x)=S_{i}(E, x)-j_{i}(E, x) / \Lambda_{i}(E)
$$

Here, $\Lambda_{\mathfrak{i}}$ is the loss mean free path which is related to the interaction mean free path, $\lambda_{i}$, by the equation

$$
1 / \Lambda_{i}=\left(1-P_{i i}\right) / \lambda_{i}
$$

where $p_{i i}$ is the average number of particles of type $i$ formed in the interaction of a type $i$ nucleus. $S_{i}$, in turn, is given by the relation

$$
s_{i}(E, x)=\sum_{k>i}^{i} j_{k}(E, x) / \Lambda_{k i}(E)
$$

where $\Lambda_{k i}$ is the mean free path for production of i-type particles from $k$-type particles. The sum in Eq. (4) can be restricted to particles 
heavier than $i$, because the lighter ones will not contribute to $i$ nuclei in an interaction, and the $i$ to $i$ type interaction is already included in the last term of Eq. (2).

Substituting Eq. (2) into (1) and rearranging terms yields

$$
\frac{d}{d x}\left[w_{i} j_{i}\right]+w_{i} j_{i} 1 / \Lambda_{i}=w_{i} s_{i}
$$

Multiplying Eq. (5) by the integrating factor $\exp (x / \Lambda)$ and rewriting the left hand side yields

$$
\frac{d}{d x}\left[\exp \left(x / \Lambda_{i}(E)\right) j_{i}(E, x) w_{i}(E)\right]=\exp \left(x / \Lambda_{i}(E) j w_{i}(E) s_{i}(E, x)\right.
$$

Eq. (b) can be used to propagate the particle energy spectra at the source, $j_{i}(E, O)$, through interstellar matter in small steps which, in the case of the calculation performed here, were $0.02 \mathrm{~g} / \mathrm{cm}^{2}$ of interstellar matter. This step size is sufficiently small that the variations in terms such as $w_{i}(E)$ and $\Lambda_{i}(E)$ introduce a negligible error into the calculation. On a given step the spectrum of the heaviest species was calculated first so that the source function for subsequent species would be available. This procedure allows averaging of the source function (actually the right-hand side of Eq. (6)) over the interval to improve the accuracy of the calculation. The range-energy relation and rate of energy loss in hydrogen were taken from the work of Barkas and Berger. ${ }^{10}$

The existing information on the energy dependence of the $\Lambda_{i}(E)$ 's and $\Lambda_{\text {lik }}$ 's is relatively meager. Only a few specific cross sections are known as a function of energy and these have been summarized by Badhwar 
and Daniel. $^{11}$ An important reason for the lack of data is that, when a proton beam bombards a particular target, the fragments of interest are left at rest in the laboratory and often only rare unstable products are readily detectable. Despite reservations related to the facts that different reaction mechanisms might produce a different energy dependence of the unknown reactions and that the very low energy regions of the parameters $(\$ 70 \mathrm{MeV} /$ nucleon) are quite uncertain, the fragmentation parameters have been estimated on the basis of the existing data. The energy dependence of the most important parameters used in the calculation is shown in Fig. 1; the high-energy values which the parameters were assumed to approach are shown in Table I..$^{12-14}$ In preparing this figure and table, where data were not available, we have assumed that the tendency for the cross sections to fall to zero below some energy $E_{0}$ was a function of the charge, $Z$, and in particular the $E_{0}$ increased with increasing $Z$ and with increasing change in $Z$ from primary to secondary particle. An alternate energy dependence of $\Lambda(0 \cdot 9,3-5)$ which emphasizes the oxygen parents more strongly is also shown in Fig. 1 .

By using the calculation and the parameters discussed above, the relative abundances of various charge species can be calculated for various source spectra. As was stated previously, it will be assumed that all nuclear species have the same spectral shape at the source and differ only in intensity. Differential spectra were selected which ranged in degree of variation in energy from $1 / \mathrm{E}^{2.5}$, where $\mathrm{E}$ is the particle kinetic energy per nucleon, to curve A in Fig. 2, which represents the May 1965 spectrum of 
helium nuclei extrapolated back through $2.8 \mathrm{~g} / \mathrm{cm}^{2}$ of interstellar hydrogen. This latter form presumes the complete absence of solar modulation and therefore represents one extreme for the spectral shape.

Next it is necessary to select the relative abundances at the source. As was stated in section II, the abundances of $\mathrm{He}^{3}$ and light nuclei were chosen to be zero at the source. In order to establish the best estimate for the remainder of the abundances it is necessary to know the average amount of material traversed by the particles from the source to the earth. The best estimate now available for the amount of interstellar matter traversed comes from the relative abundance of light nuclei at high energy. Therefore, in this paper the amount of interstellar material was determined from the light to medium nuclei ratio, and then it was seen to be consistent with the uther data. In the experimental results to be presented in section $V$, it will be seen that the best estimate we obtained for the average amount of interstellar matter traversed is $2.8 \pm 0.4 \mathrm{~g} / \mathrm{cm}^{2}$. The relative abundances at the source used in the final calculation for helium, medium $(6 \leq z \leq 9)$, $(10 \leq z \leq 19)$, and $(z \geq 20)$ nuclei were $12.1,1.00,0.36$ and 0.13 , respectively.

The results of the calculations are displayed in Figs. 3 through 8 so that the role of the various parameters involved may be seen. Fig. 3 shows the 1 ight to medium ratio for various amounts of interstellar material for a particular source spectrum $\left(\mathrm{dJ} / \mathrm{dw}=\mathrm{c} / \mathrm{w}^{2.5}\right.$, where $w$ is the total energy)

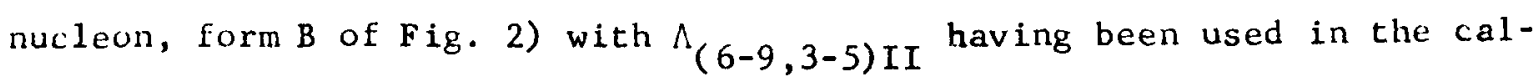
culation. The increase in this ratio as one proceeds to lower energies 
results from the combined effects of the increases in cross sections for creation of light nuclei and the differing energy loss rates of light and medium nuclei. The decrease of this ratio at very low energies arises from the decrease of the cross sections in this region. One can see that the general character of the curve is determined after about $1 \mathrm{~g} / \mathrm{cm}^{2}$ of interstellar material. Passage through additional material only serves to increase the ratio at all energies, although the increase is somewhat greater at low energies. The exact shape of the low energy end is fairly uncertain because of the lack of rather critical cross section information. Following Fig. 3, we show that the ratios of $(10 \leq Z \leq 19)$ and $(z \geq 20)$ nuclei to medium nuclei in Fig. 4 for the same source-spectral shape. Significant in this figure is the depression of the ratios at low energies after passage through small amounts of material. This effect arises because of the extremely high rate of energy loss of the heavier nuclei at low energies. Subsequently, a partial equilibrium is established in the Low energy region between loss of heavy particles via energy degradation and the supply of heavy particles from higher energies where they are now more numerous. At high energies the ratios decrease more smoothly with increasing amounts of material traversed, since here the dominant mechanism is fragmentation of the heavy nuclei. Fig. 5 shows the similar, though more marked, results using a steeper source spectrum $\left(\mathrm{dJ} / \mathrm{dE} \sim 1 / \mathrm{E}^{2.5}\right.$ ). Notice that the principal difference between Figs. 4 and 5 is in the degree of suppression of the heavier nuclei at low energies.

Continuing to examine the importance of the shape of the energy spectrum at the source, we note that Fig. 6 illustrates the light to medium 
ratio for various source spectra and the two $\Lambda_{\sigma-9,3-5)}$ functions using the best estimate of the interstellar mean free path. The effect of different source spectra on the helium to medium ratio after passage through 2.8 $\mathrm{g} / \mathrm{cm}^{2}$ is shown in Fig. 7 , where there is a marked difference in this ratio at low energies for different source spectra.

The other component of the primary cosmic radiation which is being considered in the present study is $\mathrm{He}^{3}$. In the passage of the cosmic rays through interstellar matter $\mathrm{He}^{3}$ is thought to arise predominately from collisions of $\mathrm{He}^{4}$ nuclei with hydrogen. ${ }^{11}$ This interaction leads to a $\mathrm{He}^{3} / \mathrm{He}^{4}$ ratio that is independent of energy above about $200 \mathrm{MeV} /$ nucleon and rises somewhat below this value. $11,17-20$ There is also a small contribution from the interaction of heavier elements with interstellar matter, and this effect has been taken into account in the fragmentation equations. The high energy values for the fragmentation parameters are given in Table I; ${ }^{11,14}$ these include the interactions leading to tritons because they also contribute to the $\mathrm{He}^{3}$ population through the subsequent decay of tritons into $\mathrm{He}^{3}$. The sum of these effects leads to a $\mathrm{He}^{3} / \mathrm{He}^{4}$ ratio before modulation that is estimated to be .13 at high energies for $2.8 \mathrm{~g} / \mathrm{cm}^{2}$ of interstellar matter and then rises as shown in Fig. 8 toward lower energies. The $\mathrm{He}^{3} /\left(\mathrm{He}^{3}+\mathrm{He}^{4}\right)$ ratio w111 be used for comparison with the data, since it is customarily plotted in this manner in the 1iterature. Collisions of cosmicray protons and hellum nuclei with interstellar helium nuclei may be important, but this effect is generally thought to be significant only at very low energies ( $\leq 50 \mathrm{MeV} /$ nucleon). The interaction of cosmic ray helium 
and heavy nuclei with interstellar helium should produce effects similar to their interaction with interstellar hydrogen, but considerably reduced in intensity because of che relative abundance of interstellar helium relative to hydrogen.

\section{Solar Modulation}

Between the time the cosmic rays enter the solar system and the time they reach the earth, they are modulated electromagnetically. This effect has been clearly established in recent years by an examination of the cosmic ray flux and energy spectrum as a function of the period in the solar cycle. 21-24 The modulation produces a decrease in particle intensity which is probably both rigidity and velocity dependent and this modulation may also reduce the energy of the particles. Numerous theories have been put forth to try to explain this modulation and several papers now $21-24$ exist which review and analyze the models in sufficient detail that it is not necessary to do so here. Thus, only a general discussion of the problem will be presented with specific details given only as necessary to the development of the discussion.

All of the major models proposed, such as the solar wind model, 15,25,26 the solar dipole model, ${ }^{27}$ and the electric deceleration model, ${ }^{28,29}$ depend on electric and magnetic fields either individually or together; hence, the modulation depends only on the velocity of the particle and its charge to mass ratio. Therefore, the fluxes of particles of the same velocity and charge to mass ratio are modulated in the same manner, and the relative 
abundances as a function of energy derived in the previous section will remain unchanged for these particles except that a given group of particles might be shifted to a lower energy.

If two particles have a slightly different charge to mass ratio, they will be decelerated by somewhat different amounts in terms of energy/nucleon, thereby possibly altering the ratio of two charged particles groups. Further, in the solar wind theory, for a given velocity, the flux of particles with the smaller charge to mass ratio (or greater rigidity) will be depressed least. In this latter case, the iight to medium nuclei value will be enhanced in the region of the energy spectrum where this effect is important since the average charge to mass ratio (Z/M) for light nuclei is .44 , as compared to .50 for medium nuclei. Similar considerations apply to the $(z \geq 20)$ nuclei to medium nuclei ratio, since the average charge to mass ratio for $(z \geq 20)$ nuclei is about .45 . Conversely the $\mathrm{He}^{3} /\left(\mathrm{He}^{3}+\mathrm{He}^{4}\right)$ value will be depressed. These last two points have been discussed by Hildebrand and Silberberg. ${ }^{30}$ The exact amount of enhancement or depression depends on the specific theory, and no exact theory yet exists. It is true, however, that in the solar wind theory the effect should probably be greatest in the energy interval from about 0.1 to 1.0 $\mathrm{BeV} /$ nucleon during high solar activity, and in a lower energy region near solar minimum.

Returning now to the question of deceleration, the electric deceleration model will be considered first. This theory was originally suggested by Nagashime 28 and developed further by Ehmert. 29 In this process it is assumed that a potential can be defined. Then, the observed flux can be 
calculated immediately relative to the flux outside the solar system using the extended Liouville theorem. Comparisons of the prediction of this theory to experimental observations have been made on several occasions. ${ }^{31-33}$ The present evidence seems to indicate that fair, but not perfect, agreement could be obtained for protons, helium, medium, and heavy nuclei for a mean free path of about $3 \mathrm{~g} / \mathrm{cm}^{2}$ and a source spectrum similar to curve B or C of Fig. 2. However, there are a number of difficulties with this model. Firstly, with the solar plasma density which exists, it is difficult to understand how a potential of the order of that needed, namely 0.2 to $1.0 \mathrm{BV}$, could exist at the earth relative to interstellar space. Secondly, if such a potential existed, it would accelerate electrons, and there is no experimental evidence from the study of electrons to indicate that this has occurred. 34 In fact, the work of Abraham et al. ${ }^{34}$ indicates that if there has been any acceleration of the electrons at all, it has not exceeded about $1.5 \mathrm{MeV}$. Thirdly, if such a potential existed, the decrease in the light to medium nuclei ratio below about 100 to $150 \mathrm{MeV} /$ nucleon would disappear, because this effect was due to the decrease in cross sections for the production of light nuclei below these energies in interstellar space, and these particles cannot reach the earth if there is a potential that large. The rise in the light to medium nuclei ratio due to the increase in the appropriate cross section values at intermediate energies combined with a small, but contributing modulation effect would then lead to a light to medium nuclei ratio which continues to rise toward lower energies--contrary to observation--as will be seen in the 
next section. One final difficulty is that the source spectrum which would give the best agreement between the proton and alpha data in the electric deceleration model is too steep to agree with the heavy to mediun muclei ratio, if it is assumed that all source spectra have the same shape.

As was mentioned earlier, there could be other types of deceleration such as the inverse betatron effect ${ }^{15}$ or the Fermi mechanism 1,2 which would decelerate both nuclei and electrons. Most estimates suggest that these effects are small; however, rarker ${ }^{15}$ has shown that the betatron effect might be important. With either of these latter mechanisms, the first objection listed before is naturally renoved since large potentials over great regions of space are not required. With respect to the second earlier objection, electrons would now be decelerated as well as protons, rather than accelerated. Existing radio astronomical data, though inconclusive, suggest that electrons have nut been severly decelerated: such deceleration would imply that the flux of high energy electrons is much larger outside the solar system than near the earth, and has a steeper spectrum at a few hundred MeV. However, if the low energy (1-10 MeV) electrons have a higher velocity than protons of the same energy, and a much lower rigidity--perhaps small enough that they can virtually follow the field lines into the solar system without being appreciably scattered--they may not be appreciably decelerated because, as rarker pointed out, the degree of deceleration in the betatron case, and in most models based on the Fermi mechanism, depends on the time spent in the solar system. 
The considerations with respect to the light to medium nuclei ratio remain, but these cannot eliminate the possibility of a small deceleration. The degree of deceleration, especially at low solar activity when the relevant data were obtained, must then be relatively sma11, and the relation between the proton and helium spectra probably must be explained in another way than by appreciable deceleration in the solar system.

It is still possible that there may be some deceleration of the cosmic rays by the solar system during periods of high solar activity. An interesting test would be the observation of a shift in the peak of the light to medium nuclei ratio to lower energy as solar activity increases. Good spectral data would be needed to separate deceleration effects from depressive rigidity dependent effects, but by studying other ratios, such as $\mathrm{He}^{3} /\left(\mathrm{He}^{3}+\mathrm{He}^{4}\right)$ and protons to helium nuclei, it should be possible to do so.

\section{Discussion}

\section{Comparison with Experimental Data}

Having discussed the interstellar travel of cosmic radiation and the solar modulation, it is now appropriate to compare the predictions of the theories to the experimental results--making a particular effort to separate the effects of the two phenomena wherever possible. In what follows the experimental data will be examined from the viewpoint of investigating the solar modulation, interstellar travel, and the original source spectra and composition.

In the last section it was demonstrated that the solar modulation probably depends only on the velocity and the charge to mass ratios of the particles. Since, fluxes of particles with the same velocity and charge to 
mass ratio would be reduced by the same amount, leaving the relative abundances unchanged. These same relative abundances as a function of energy/nucleon therefore would also exist outside the solar system and be directly comparable to those calculated in section III. Figs. 9 and 10 show the ratios of $(z \geq 10)$ nuclei and $(z \geq 20)$ nuclei to medium nuclei as measured by several experimenters. Since these ratios seen independent of the period in the solar cycle, no attempt has been made to separate the measurements into different time periods. In fact, the very heavy to medium ratio might be expected to vary somewhat with time in the solar cycle because of the difference in the modulation effect resulting from the small difference in the charge to mass ratio mentioned in the previous section. The curves for the predicted ratios as a function of energy for $2.8 \mathrm{~g} / \mathrm{cm}^{2}$ of interstellar material and several different source spectra are also shown in Figs. 9 and 10 . There is generally good agreement between the experimental values and the calculated curves. No real selection of source spectra can be made from these data alone with the exception of rejecting very steep source spectra of the form of Curve $D$ in Fig. 2 or steeper.

The similar data for the helium to medium ratio are shown in Fig. 11 . In order that there be agreement between the calculated curves and the data in this case, the source spectrum must be quite flat--approximately of the form of curve A or B in Fig. 2 Here again it has been assumed that the cosmic radiation has passed through approximately $2.8 \mathrm{~g} / \mathrm{cm}^{2}$ of interstellar material, as the relative abundances of the light nuclei indicate. As has been mentioned, a small error in the mean free path $\left( \pm 1 \mathrm{~g} / \mathrm{cm}^{2}\right)$ does not 
affect this conclusion because of the relatively slow variation of the curve for the medium to helium nuclei ratio with interstellar path length after the first $\mathrm{g} / \mathrm{cm}^{2}$.

In Fig. 12, the experimental data for the $(Z \geq 20)$ to helium nuclei flux ratios are compared to the predicted curves. The somewhat poorer agreement between the predicted curves and observed points in Fig. 12 seems to be due $p$ rimarily to differences in experimental data--that is, higher values for the $(\% \geq 20)$ to helium nuclei ratio than the directly measured values ut reference 51 are obtained at high energies by taking ratios of the experimental data presented in the two pervious figures.

The light to medium ratio has a more complicated energy dependence as was shown in Fig. 6. The experimental data for the lighi to medium ratio are compared to the predicted values for $2.5,2.8$, and $3.1 \mathrm{~g} / \mathrm{cm}^{2}$ of interstellar matter in Fig. 13 for a spectrum of the form of curve $B$ of Fig. 2. The reason for choosing approximateiy $2.8 \mathrm{~g} / \mathrm{cm}^{2}$ for the interstellar path length is now, of course, clear from an examination of the high energy region of the curve, where the ratio is independent of spectral shape. Notice that there is reasonable agreement in the energy range from 150 to $1000 \mathrm{MeV}$ / nucleon, especially since the modulation effect will also probably increase the light to medium nuclei ratio slightly in this region, whereas at high and possibly very low energies it will not. Estimates ${ }^{16}$ of the importance of this effect indicate that it might cause the 1 ight to medium nuclei flux ratio to increase by from 0 to 25 percent over the values in Fig. 13 in the energy region around $300 \mathrm{MeV} /$ nucleon where the effect is thought to be most pronounced during the period when the measurements were made. Later in 
this section, we shall discuss reasons for believing the increase is neariy zero near solar minimum.

In the low energy region there is not enough information to determine the variation with energy of the cross sections leading to the production of light nuclei. If the approach is assumed to be correct, the results would indicate the cross sections were closer to the type II cross sections than to the type I values. Since the type II cross sections emphasize oxygen this result is in agreement with the conclusions of Reames and Fichte ${ }^{45}$ which show oxygen to be more abundant than carbon (especially at the source). These data also argue against steep source spectra which yield large light to medium nuclei ratios at iow energies (see Fig. 6).

Looking next at the $\mathrm{He}^{3} / \mathrm{He}$ results, Fig. 14 shows that, with the exception of three points, the experimental data seem to agree well with the curve which represents the expected ratio for a source spectrum of the form of $A$ or $B$ in $F i g . ~ 2$ and $2.8 \mathrm{~g} / \mathrm{cm}^{2}$ of interstellar material. since the three data points at higher energies which disagree were taken at times close to the other experiments, thi discrepancy must be considered as one between experiments rather than between calculated and observed values. The recent experiments which have resolution and record the particles themselves directly all agree with the curve. Thus, until there is further evidence to the contrary, we shall assume the data points agree with the curve. This is consistent with the previous discussion of a relatively flat source spectrum and little mudulation near solar minimum. In the low energy region, modulation would reduce the $\mathrm{He}^{3} / \mathrm{He}$ ratio. $\Lambda s$ was indicated before, it is difficult to know the degree of the suppres sion produced by the modulation; however, it would be a larger effect than 
that for the light to medium nuclei ratio because of the larger difference in the charge to mass ratio. The $\mathrm{He}^{3} / \mathrm{He}$ ratio, therefore, represents an interesting test of rigidity dependent solar modulation effects, and it can be used throughout the solar cycle as a check for the conclusions deduced from proton and helium studies.

\section{Degree of Solar Modulation}

The considerations presented in section IV indicated already that there is no appreciable deceleration of the cosmic rays near sular mininum, but the question of whether the depression of the cosmic ray flux is or is not appreciable has yet to be considered. Earlier in this section it was seen that, if the average interstellar path length of $2.8 \mathrm{~g} / \mathrm{cm}^{2}--$ deduced from the 1 ight to medium nuclei ratio--is used, the observed relative abundances of He, medium, and $(10 \leq z \leq 19)$ nuclei can be produced fur similar source spectra only if the source spectrum is very nearly that which will give the cosmic-ray-maximum spectra after passage through 2.8 $\mathrm{g} / \mathrm{cm}^{2}$, that is, specifically of the form of curves $A$ or $B$ in Fig. 2 . The observed very heavy to medium nuclei ratio is also consistent with this type of source spectrum; but this is a slightly less severe test because the latter ratio could, in principle, be enhanced by modulation at low energies since the very heavy nuclei have slightly smaller charge to mass ratios than the medium nuclei. With the stated assumption, the data on relative abundances mentioned above imply that the cosmic radiation is only very slightly modulated when it reaches its maximum value near solar minimum.

If there is in fact very little or no modulation near solar minimum, then the proton and helium nuclei apparently have very different energy: 
nucleon spectra. This statement follows from the fact that if they have the same energy/nucleon spectra a very large modulation is needed to produce the ubserved proton to helium nuclei ratio as a function of energy. It is also unlikely that the rigidity spectra of the protons and helium nuclei are similar at the source under the same assumptions if the data are correct because the source spectra predicted for helium nuclei would then be of the form of curve $C$ in Fig. $2 .^{16}$ An alternate possibility is that the proton to helium nuciei ratio is in fact about five for the same energy/nucleon as observed at low energies, and the flux measurements at high energies have been in error in that a satisfactory albedo correction has not been made. Analysis of the albedo correction made by McDonald 66 makes the latter alternative seem unlikely.

An acceleration mechanism that is both velocity and rigidity dependent, such as a modified Fermi mechanism ${ }^{6,67,68}$ could produce different source energy/nucleon spectra for particles with different $Z / M$ values such as helium nuclei and protons. An example of this type of acceleration occurring in nature is the solar cosmic ray case. If such a difference exists between proton and helium nuclei spectra, a difference would be expected in the helium and very heavy nuclei spectra also, but it would be small because of the small difference in charge to mass ratio. This feature may explain the tendency of the predicted ratio for $(z \geq 20)$ nuclei to helium nuclei to fall slightly above the observed values at high energy in Fig. 12. It shall be remembered that both $\mathrm{He}^{3}$ and $\mathrm{light}$ nuclei are assumed to be secondaries formed in interactions in interstellar space; so their energy 
spectra presumably reflect those of their parents except for the effect of different rates of energy loss as discussed in section III.

\section{Other Theoretical Interpretations}

There are a number of other possible theoretical approaches related to the study of cosmic radiation which in general are more complex and involve more adjustable parameters and assumptions. We shall now examine a few of the more prominent of these in the light of what has been discussed.

The first is the possibility of varying path lengths for different particles. Since the travel of cosmic rays is probably a diffusion process, some cosmic rays may have traversed more material than others. Balasubrahmanyan et al. ${ }^{69}$ have treated this problem recently in some detail. An examination of the results presented here for path lengths from 1 to $6 \mathrm{~g} / \mathrm{cm}^{2}$ indicates that the variation of ratios and fluxes is sufficiently slow and smooth that the conclusions reached on the basis of assuming all cosmic rays will have traversed a given amount of material, $x_{0}$, will be essentially the same as assuming a reasonable distribution about an average value of $\mathrm{x}_{0}$. Hence, the conclusions will be the same. Another possibility is that the average path length varies with the energy of the particle. Apparao ${ }^{70,71}$ has considered the effect of this possibility and gives the necessary form for the variation to obtain agreement with some of the experimental data under the assumption that the cross sections (or fragmentation parameters)are independent of energy and the source spectrum is of the form $c / w^{\gamma+1}$. There seem to be several detail 
difficulties, but the most striking disagreement is in the low energy light to medium data recently obtained and shown in Fig. 12.

Kan'lon and Skadron ${ }^{72}$ have pointed out that agreement can be obtained with the experimental data for the light to medium ratio if the path length Lirst incuates with energy to about $200 \mathrm{MeV} /$ nucleon and then decreases and finaliy approaches a constant at high energies. They suggest that this encrgy dependence of the path length may result from a rigidity dependent escape mechanism at the source. The necessity of this assumption secms to be eliminated when the variation of the interaction mean Lee paths with energy is considered.

finother aproach to an explanation of the observed relative abundances has been presented by Lim and Fukui. ${ }^{47}$ They point out that the heavy to nedium and medium to helium ratios could be explained by a large residual deceleration in the solar modulation. There remains, however, the difliculty of the light to medium nuclei ratio at low energies in that case, as well as the other problems mentioned in the modulation section. Coustock, Fan, and Simpson ${ }^{38}$ have reemphasized that the relative abundance ul light nuclei relative to heavier ones has been the principal reason tor assuming the cusmic rays have passed through about $2.8 \mathrm{~g} / \mathrm{cm}^{2}$, and, if, on the uther hand, the cosmic rays have passed through almost no material, the relative abundances of helium, medium and heavier nuclei would remain virtually independent of energy, as is observed. An examination of the electron data ${ }^{73}$ also suggests the possibility that the cosmic rays may have passed through very little material. Therefore, if there is sume reasonable way of explaining the presence of 1 ight nuclei and $\mathrm{He}^{3}$ in the source 
region before acceleration with their particular relative abundances as a function of energy, the study of interstellar travel can be reexamined without the restriction of a path length of several $\mathrm{g} / \mathrm{cm}^{2}$. This suggestion--although an interesting possibility which should not be completely ignored--presently seems much less likely than the formation of light nuclei in collisions in interstellar space for the reasons stated in section II of this paper.

Finally, some data ${ }^{74}$ seem to indicate that the helium to medium nuclei ratio may increase again at high energies ( $\geq 5 \mathrm{BeV} /$ nucleon). It has been suggested by Apparao ${ }^{71}$ and Webber ${ }^{74}$ that this characteristic, may be due to a difference in the energy spectra of helium and medium nuclei. There is no evidence to indicate that the cross sections for medium nuclei incident upon hydrogen increase appreciably at high energies, or that the cross section for helium nuclei incident upon hydrogen decreases appreciably. Thus, if the helium to medium nuclei ratio in the region from 5 to $50 \mathrm{BeV} /$ nucleon should prove larger than that in the .5 to $5 \mathrm{BeV} / \mathrm{nucleon}$ region, a real difference in source spectra would apparently be indicated. If this difference is accepted, then virtually any model will give satisfactory agreement with experiments for some set of source spectra. Since at this time there is no compelling reason to choose any particular set of relative source spectra other than assuming all particles with the same charge to mass ratio have the same energy/nucleon spectra as chosen here, this possibility will not be explored further. 
VI. Conclusion

The analysis which has been presented here shows that the experimental data on the multiply charged cosmic ray nuclei seem to be in generaliy good agreement with the predictions on the simple set of assumitjuns made at the beginning of the article. As a reminder, these were:

(a) All muitiply charged cosmic ray nuclei have the same spectral shape at the source.

(b) The relative abundances of $\mathrm{He}^{3}$ and 1 ight nuclei at the source are negligibly small compared to those of $\mathrm{He}^{4}$ and medium nuclei respectively.

(،) The average amount of interstellar matter traversed is independent of energy.

With these assumptions it is possible to obtain reasonably good agreement with the experimental data on the relative abundances for the multiply charged nuclei under the following conditions:

(a) The difterential energy spectra at the suurce must be quite llat of the form of curve $A$ in Fig. 2 or possibly curve $B$, but not much steeper than $B$ and certainly not as steep as curve $C$. This condition is set particularly strongly by the helium to medium nuclei flux ratio as a function of energy.

(b) The average interstellar path length is $2.8 \pm 0.4 \mathrm{~g} / \mathrm{cm}^{2}$.

(c) The relative abundances of the helium, medium, ( $10 \leq 7 \leq 19)$ and $(\% \geq 20)$ nuclei at the source are approximately $12.1,1.00,0.36$, and 0.13 . The uncertainty of these numbers is probably between $5 \%$ and $10 \%$ if the assumptions are valid. 
These results together with the data on the electrons also imply the following:

(a) There is little or no deceleration in the solar modulation mechanism at least near solar minimum.

(b) There is very little modulation of the cosmic ray spectra near solar minimum, since curve A in Fig. 2 is in fact the cosmic ray helium nuclei maximum spectrum extrapolated back to the source.

(c) Assuming the observational data to be correct, protons and helium nuciei have different energy/nucleon spectra at the source. This conclusion is a corollary of the preceding conclusion because the marked difference observed in the cosmic ray proton and helium nuclei spectra inust then reflect differences at the source, since only a very large modulation effect could explain the different spectra otherwise.

There are also several postulates which have been suggested which are apparently not necessary in any marked degree to explain the cosmic ray data. For example, it is not necessary to assume an energy dependent average mean free path in interstellar matter. There is also no need to try to explain the relative abundances as a function of energy, and in particular the abundances of helium, medium, and heavier nuclei, by assuming that light nuclei and $\mathrm{He}^{3}$ are formed in some other way than by fragmentation. This result follows from the fact that it is possible to reproduce observed relative abundances from similar source spectra even after passage through $2.8 \pm 0.4 \mathrm{~g} / \mathrm{cm}^{2}$ of interstellar matter. Finally, it is, at least present1y, not necessary to assume differences in the energy spectra of the multiply charged nuclei to explain the data, although refined data may indicate small differences at high energies. 
Hence, with the simple stated assumptions, it seems possible at present to explain the existing cosmic ray data and at the same time possibly to gain some insight into the solar modulation mechanism, the average amount of material through which cusmic rays have passed, the source spectrum of the multiply charged cosmic rays, and the relative abundances of cosmic ray multiply charged nuciei at the suurce.

Acknowiodgment:

We are happy to acknowledge the heipful comments of Dr Frank $\mathrm{B}$ McDunaid. 


\section{FIGURE CAPTIONS}

Fig. 1. Variation of various mean free paths with energy deduced from the experimental data summarized by badhwar and Daniel. 11 See the text for a discussion of these curves. The numbers in the subscripts of $\Lambda_{(i, j)}$ in the figure refer to the charge interval.

Fig. 2. Some of the source spectra assumed for the calculations in this paper. "A" represents the May 1965 cosmic ray helium nuclei spectrum extrapolated back to the source; "B" is the curve $0.6 / W_{N}{ }^{2.5}$, where $W_{N}$ is the total energy in BeV/nucleon; "C" is the spectrum which both the protons and helium nuclei could have if they had similar rigidity spectra at the source and underwent a diffusion-convection type modulation ${ }^{5}$ within the solar system ${ }^{6}$; and "D" is the curve $107 / \mathrm{E}_{\mathrm{N}}{ }^{2.5}$, where $E_{\mathrm{N}}$ is the kinetic energy in $\mathrm{MeV} / \mathrm{nucleon}$.

Fig. 3. The calculated light to medium nuclei ratio as a function of energy for a source spectrum of the form of curve $B$ in Fig. $2,1 / \Lambda_{(f,-9,3-\infty) I I}$, and the mean free paths indicated in the figure.

Fig. 4. Variation of the calculated $(10 \leq Z \leq 19)$ and $(Z \leq 20)$ to medium nuclei ratios with energy/nucleon for a source spectrum of the form of curve B in Fig. 2 and the mean free paths indicated in the figure.

Fig. 5. Variation of the calculated $(10 \leq Z \leq 19)$ and $(Z \leq 20)$ to medium nuclei ratios with energy/nucleon for a source spectrum of the form of curve D in Fig. 2 and the mean free paths indicated in the figure. 
Fig. 6. Variation of the calculated light to medium nuclei ratio for the four energy/nucleon spectra of Fig. 2 (1abeled A through D), the two types of fragmentation parameters considered (1abeled I and II), and interstellar mean free paths of 1.4 and $2.8 \mathrm{~g} / \mathrm{cm}^{2}$.

Fig. 7. Variation of the calculated helium to medium nuclei ratio with energy/nucleon for the four source spectra of Fig. 2 (labeled A through D), and interstellar mean free paths of $0.0,0.5$, and $2.8 \mathrm{~g} / \mathrm{cm}^{2}$.

Fig. 8. Variation of the calculated $\mathrm{He}^{3} / \mathrm{He}^{4}$ ratio with energy/nucleon for the four source spectra of Fig. 2 (labeled A through D), and interstellar mean free paths of $1.0,2.0$, and $2.8 \mathrm{~g} / \mathrm{cm}^{2}$.

Fig. 9. Comparison of the experimental data with the calculated variation with energy/nucleon of the $(Z \leq 10)$ to medium nuclei ratio for an interstellar mean free path of $2.8 \mathrm{~g} / \mathrm{cm}^{2}$. Letters refer to the source spectra in Fig. 2, and numbers by the experimental points give the references.

Fig. 10. Comparison of the experimental data with the calculated variation with energy/nucleon of the $(Z \leq 20)$ to medium nuclei ratio for an interstellar mean free path of $2.8 \mathrm{~g} / \mathrm{cm}^{2}$ and $(Z<20)$ to medium nuclei ratios at the source of 0.125 (solid curves) and 0.15 (dashed curve). Letters refer to the source spectra in Fig. 2, and numbers by the experimental points give the references. 
Fig. 11. Comparison of the experimental data with the calculated variation with energy/nucleon of the helium to medium nuclei ratio for an interstellar mean free path of $2.8 \mathrm{~g} / \mathrm{cm}^{2}$. Letters refer to the source spectra in Fig. 2, and numbers by the experimental points give the references. The uncertainty in the ratio of reference 38 may be larger than indicated because the majority of the medium nuclei were in a higher energy/nucleon interval than the helium nuclei, and, therefore, a knowledge of the spectral shape is assumed.

Fig. 12. Comparison of the experimental data with the calculated variation with energy/nucleon of the $(z \geq 20)$ to helium nuclei ratio for an interstellar mean free path of $2.8 \mathrm{~g} / \mathrm{cm}^{2}$, and $(z \geq 20)$ to helium nuclei ratios at the source of $1.05 \times 10^{-2}$ (solid curves) and $1.25 \times 10^{-2}$ (dashed curve). Letters refer to the source spectra in Fig. 2, and numbers by the experimental points give the references. The uncertainty in the ratio of reference 38 may be larger than indicated because the majority of the $(\mathrm{Z} \geq 20)$ nuclei were in a higher energy/nucleon interval than the helium nuclei, and, therefore, a knowledge of the spectral shape is assumed.

Fig. 13. Comparison of the experimental data with the calculated variation with energy/nucleon of the light to medium nuclei ratio for interstellar mean free paths of $2.5(\cdot-), 2.8 \leftrightarrow$, and $3.1(--)$ $\mathrm{g} / \mathrm{cm}^{2}$ and $\Lambda_{(6-9,3-5) I I}$. Letters refer to the source spectra in Fig. 2, and numoers by the experimental points give the references. 
Fig. 14. Comparison of the experimental data with the calculated variation with energy/nucleon of the $\mathrm{He}^{3}$ to $\left(\mathrm{He}^{3}+\mathrm{He}^{4}\right)$ nuclei ratio for an interstellar path of $2.8 \mathrm{~g} / \mathrm{cm}^{2}$. Letters refer to the source spectra in Fig. 2, and numbers by the experimental points give the references. 
Table I

Asympotic Fragmentation Parameters and Interaction Mean Free Paths

(a) High Energy ( $\div-1 \mathrm{BeV} /$ nucleon) Fragmentation parameters (the average number of secondaries of type $j$ produced by a primary of type $i$ ) in hydrogen, from the summary and calculations of Badhwar et a $1^{12}$ and Kaplon and Skadron ${ }^{14}$.

Primary

$\begin{array}{lcccccc} & (20 \leq \mathrm{Z} \leq 30) & (10 \leq \mathrm{Z}=19) & (6 \div \mathrm{Z} \leq 9) & (3 \leq \mathrm{Z} \leq 5) & \mathrm{He}^{4} & \mathrm{He}^{3} \\ (20 \leq \mathrm{Z} \leq 30) & .22 & .06 & .03 & .05 & .49 & .15 \\ (10 \leq \mathrm{Z} \leq 19) & & .17 & .07 & .06 & .30 & .15 \\ (6 \leq \mathrm{Z} \div 9) & & .11 & .08 & .20 & .20 \\ (3 \leq \mathrm{Z} \leq 5) & & & .08 & .13 & .20 \\ \mathrm{He}^{4} & & & & .06 & .40\end{array}$

(b) Interaction Mean Free Paths in hydrogen ${ }^{12}$ at high energies ( $\mathrm{E} \geq 1 \mathrm{BeV} / \mathrm{nucleon}$ Charge $\quad(20 \leq Z \leq 30) \quad(10 \leq Z \leq 19) \quad(6 \leq Z \leq 9) \quad(3 \leq Z \leq 5) \quad 2$

$\begin{array}{llllll}\therefore\left(\mathrm{g} / \mathrm{cm}^{2}\right) & 2.6 & 4.2 & 6.3 & 9.8 & 14.6\end{array}$


1. E. Fermi, Phys. Rev. 75, 1169 (1949).

2. E. Fermi, Astrophys. Jour. 119, 1 (1954).

3. \$. I. Syrovatskii, Soviet Phys. JETP 40, 1788 (1961).

4. S. A. Colgate, and H. J. Johnson, Phys. Rev. Letters 5, 235 (1960).

5. S. A. Colgate, Paper Accel 4, Ninth International Conference on

Cosmic Rays, London, Sept. 1965.

6. S. Biswas, C. E. Fichte1, and D. E. Guss, Phys. Rev. 128, 2756 (1962).

7. S. Biswas, C. E. Fichtel, D. E. Guss, and C. J. Waddington J. Geophys. Res. 63, 3109 (1963).

8. H. E. Suess and H. C. Urey, Reviews of Modern Physics 28, 53 (1956).

9. E. C. Ray, J. Geophys. Res., 65, 1126 (1960).

10. W. H. Barkas and M. J. Berger, National Academy of Sciences National Research Council Publication 1133, 103 (1964).

11. G. D. Badhwar, and R. R. Daniel, Progress of Theoretical Physics $\underline{30}$, 615 (1963).

12. G. D. Badhwar, R. R. Daniel, and B. Vijayalakshmi, Progress of Theoretical Physics 28, 607 (1962).

13. M. W. Friedlander, K. A. Neelakantan, S. Tokunaga, G. R. Stevenson, and C. J. Waddington, Phi1. Mag. $\underline{8}$, No. 94, 1691 (1963).

14. M. F. Kaplon and G. Skadron, Nuovo Cimento 34, 1687 (1964).

15. E. N. Parker, Interplanetary Dynamic Processes, Interscience Publishers (1963).

16. N. Durgaprasad, C. E. Fichtel, and D. E. Guss, Ninth International Conference on Cosmic Rays, London, Spec. 19 (1965). 
17. A. F. Vickersham, Phys. Rev., 107, 1050 (1957).

18. P. Tenneswald, Phys. Rev. 89, 508 (1958).

19. M. S. Kosodaev, M. M. Kulyukin, R. M. Sulyaev, A. I. Fillipov, and Y. A. Shcherbahov, JETP 2, No. 3, 511 (1960).

20. W. H. Innes, University of California Radiation Laboratory Report UCRL-8040 (1957) (unpublished).

21. W. R. Webber, Progress in Elementary Particles and Cosmic Ray Physics VII, North Holland Publishing Company, Amsterdam $\underline{6}, 75$ (1963).

22. L. I. Dorman, Progress in Elementary Particles and Cosmic Ray Physics VII, North Holland Publishing Company, Amsterdam 6, 75 (1963).

23. W. R. Webber, and F. B. McDonald, J. Geophys. Res. 69, 3097 (1964).

24. C. E. Fichtel, D. E. Guss, G. R. Stevenson, and C. J. Waddington, Phys. Rev. 133, B818 (1964).

25. E. N. Parker, Phys. Rev. 109, 1874 (1958).

26. E. N. Parker, Phys. Rev. 110, 1445 (1958).

27. H. E1liot, Phil. Mag. E, 601 (1960).

28. K. Nagashima, J. Geomag. and Geoelec. 3, 100 (1951).

29. A. Ehmert, Proc. Moscow Cosmic Ray Conf. IV, 142 (1960).

30. B. Hildebrand, and R. Silberberg, Regional IQSY Symposium, Buenos Aires (1964).

31. F. B. McDonald, Nuovo Cimento, Suppl 2 , 500 (1958).

32. C. E. Fichte1, Nuovo Cimento 19, 1100 (1961).

33. P. S. Freier and C. J. Waddington, Space Science Reviews 4 , 313 (1963). 
34. P. Abraham, K. Brunstein and T. Cline, On the Production of LowEnergy Cosmic-Ray Electrons, to be published.

35. V. L. Ginzburg, and S. I. Syrovatskii, The Origin of Cosmic Rays, Macmillan Co., New York, p. 326 (1964).

36. H. Aizu, Y. Fujimoto, S. Hasegawa, M. Koshiba, I. Mito, J. Nishimura, and K. Yakoi, Supp1. Progr. Theoret. Phys. (Kyoto) 16, 54 (1960)

37. M. Koshiba, E. Lohrman, H. Aizu, and E. Tamai, Phys. Rev. 131, $2692(1963)$.

38. G. M. Comstock, C. Y. Fan and J. A. Simpson, "Abundances and Energy Spectra for Nuclei of Galactic Origin above $20 \mathrm{MaV}$ per Nucleon," Ninth International Conference on Cosmic Rays, London (1965).

39. P. S. Freier, E. P. Ney, and C. J. Waddington, Phys. Rev. 113, 921 (1959).

40. R. R. Daniel and N. Durgaprasad, J. Phys. Soc. Japan 17, Supp. A III, 15 (1962).

41. F. W. O'Del1, M. M. Shapiro, B. Stiller, J. Phys. Soc. Japan 17 , Supp. A III, 23 (1962).

42. P. L. Jain, E. Lohrmann and A. W. Tencher, Phys. Rev. 115, 654 (1959)

43. C. M. Garelli, B. Quassiati and M. Vigore, Nuovo Cimento 15, 121 (1960).

44. I. S. van Heerden and B. Judek, Can. J. Phys. 38, 964 (1960).

45. C. E. Fichtel and D. V. Reames, "Observation of the Elemental Abundances in Low-Energy Cosmic Rays in July, 1964," (to be Published).

46. C. E. Fichte1, D. E. Guss, and K. A. Neelakantan, Phys. Rev. 138 , B732 (1965). 
47. Y. K. Lim, and K. Fukui, J. Geophys. Res. 70, 4965 (1965).

48. F. B. McDonald and W. R. Webber, J.G.R. 67, 2119 (1962).

49. V. K. Balasubrahmanyan, D. E. Hagge, G. H. Ludwig, and F. B. McDonald, The Multiply-Charged Primary Cosmic Radiation at Solar Minimum, NASA $X-611-65-480(1965)$.

50. P. S. Freier, J. S. Rno, and C. J. Waddington, Ninth International Conference on Cosmic Rays, London, (1965).

51. W. R. Webber, J. F. Ormes, and Ivan Rosenvinge, Ninth International Conference on Cosmic Rays, London (1965).

52. W. R. Webber and J. Ormes, International Conference on Cosmic Rays, Jaipur (1963).

53. C. J. Waddington and P. S. Freier, Ninth International Conference on Cosmic Rays, London, (1965).

54. V. K. Balasubrahmanyan and F. B. McDonald, J.G.R. 69, 3289 (1964).

55. G. D. Badhwar, S. N. Devanathan, and M. F. Kaplon, J. Geophy - Res . 70, 1005 (1965).

56. F. Foster and A. Debenedetti, Nuovo Cimento 28, 1190 (1963).

57. M. V. K. Apparao, J. Geophys. Res. 67, 1289 (1962).

58. C. Dahanayake, M. F. Kaplon, and P. J. Lakakare, University of Rochester Report URPA-31 (1964).

59. F. Foster and J. H. Mulvey, Nuovo Cimento, 27, 93 (1963).

60. B. Hildebrand, F. W. O'Dell, M. M. Shapiro, R. Silberberg, and B. Stiller, Proceedings of the International Conference on Cosmic Rays, Jaipur $\underline{3}$, (1963).

61. H. Aizu, Proceedings of the International Conference on Cosmic Rays, Jaipur $\underline{3}, 90$ (1963). 
62. D. J. Hofmann, and J. R. Winckler, Phys. Rev. Letters 16, 109 (1966).

63. W. R. Webber, and J. F. Ormes, Phys. Rev. 138, B416 (1965).

64. F. W. O'De11, B. Hildebrand, M. M. Shapiro, R. Silberberg, and B. Stiller, The Ratio of $\mathrm{He}^{3}$ to $\mathrm{He}^{4}$ in the Primary Cosmic Radiation, Regional IQSY Symposium, Buenos Aires (1964).

65. S. Biswas, Ninth International Conference on Cosmic Rays, London (1965).

66. F. B. McDonald, Phys. Rev. 109, 1367 (1958).

67. D. G. Wentze1, J. Geophys. Res. 70, 2716 (1965).

68. S. Hayakawa, J. Nishimura, H. Obayashi, and H. Sato, Acceleration Mechanisms of Cosmic Rays, Nagoya University preprint (1965).

69. V. K. Balasubrahmanyan, E. Boldt, and R. A. R. Palmeira, Phys. Rev. 140, B1157 (1965).

70. M. V. K. Apparao, Nuovo Cimento 32, 1158 (1964).

71. K. Apparao, Proceedings of the Indian Academy of Sciences, 62,78 (1965).

72. M. F. Kaplon and G. Skadron, Reviews of Geophysics, to be published. 73. K. A. Brunstein and T. L. Cline, "Possible Neutrality Cosmic Rays," NASA $X-611-65-479(1965)$.

74. W. R. Webber, Ninth International Conference on Cosmic Rays, Lond on (1965). 


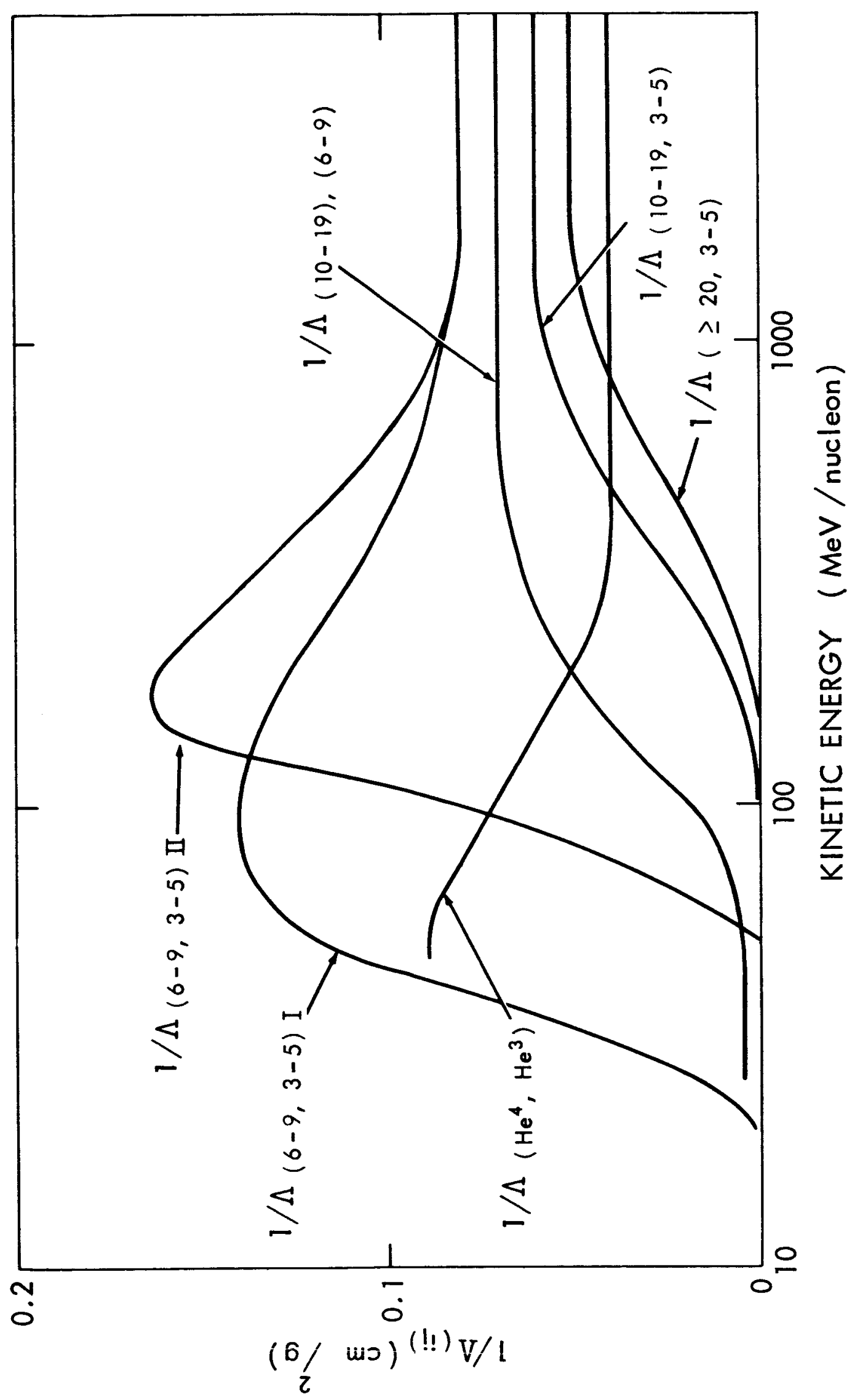




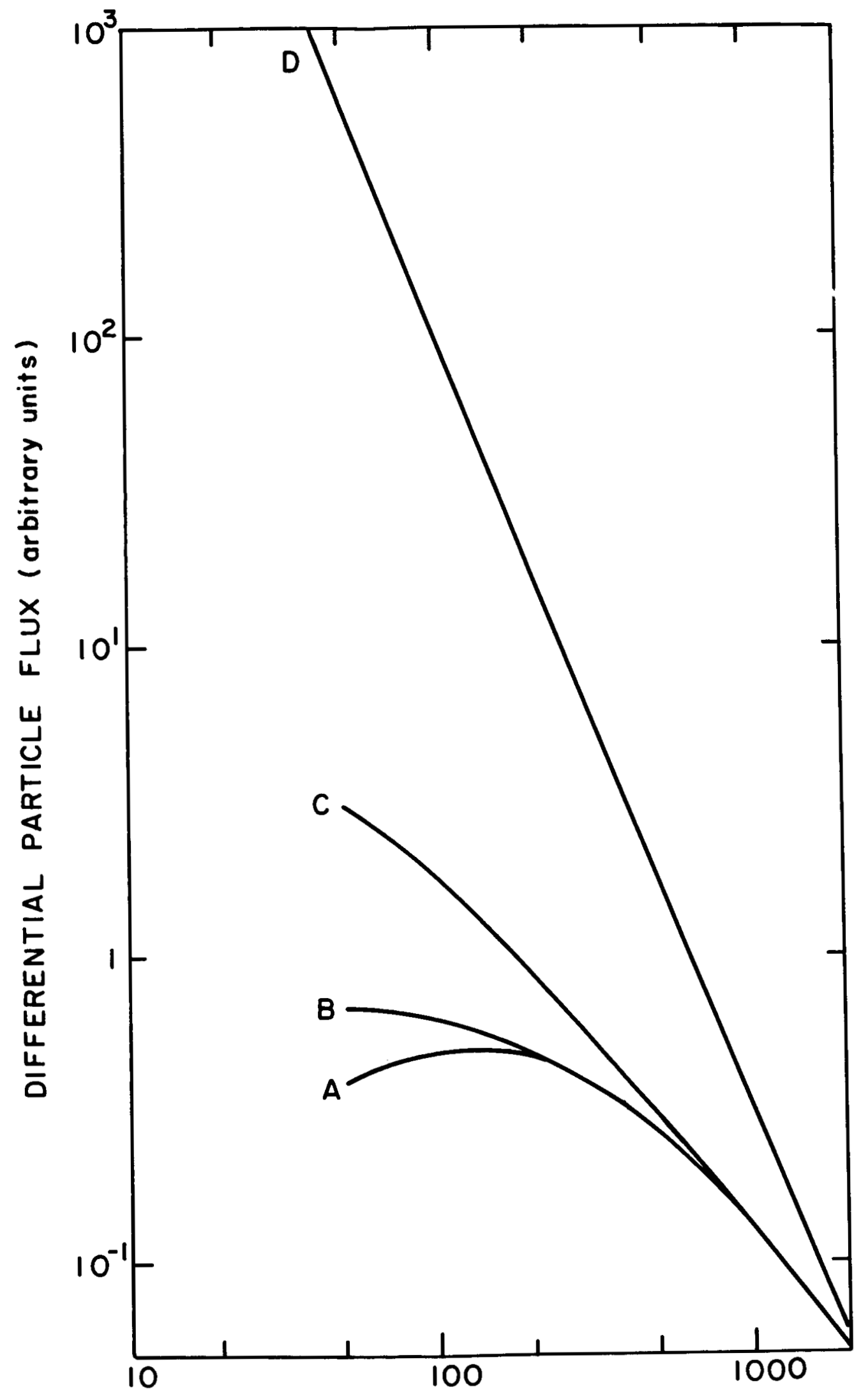

KINETIC ENERGY (MeV/nucleon) 


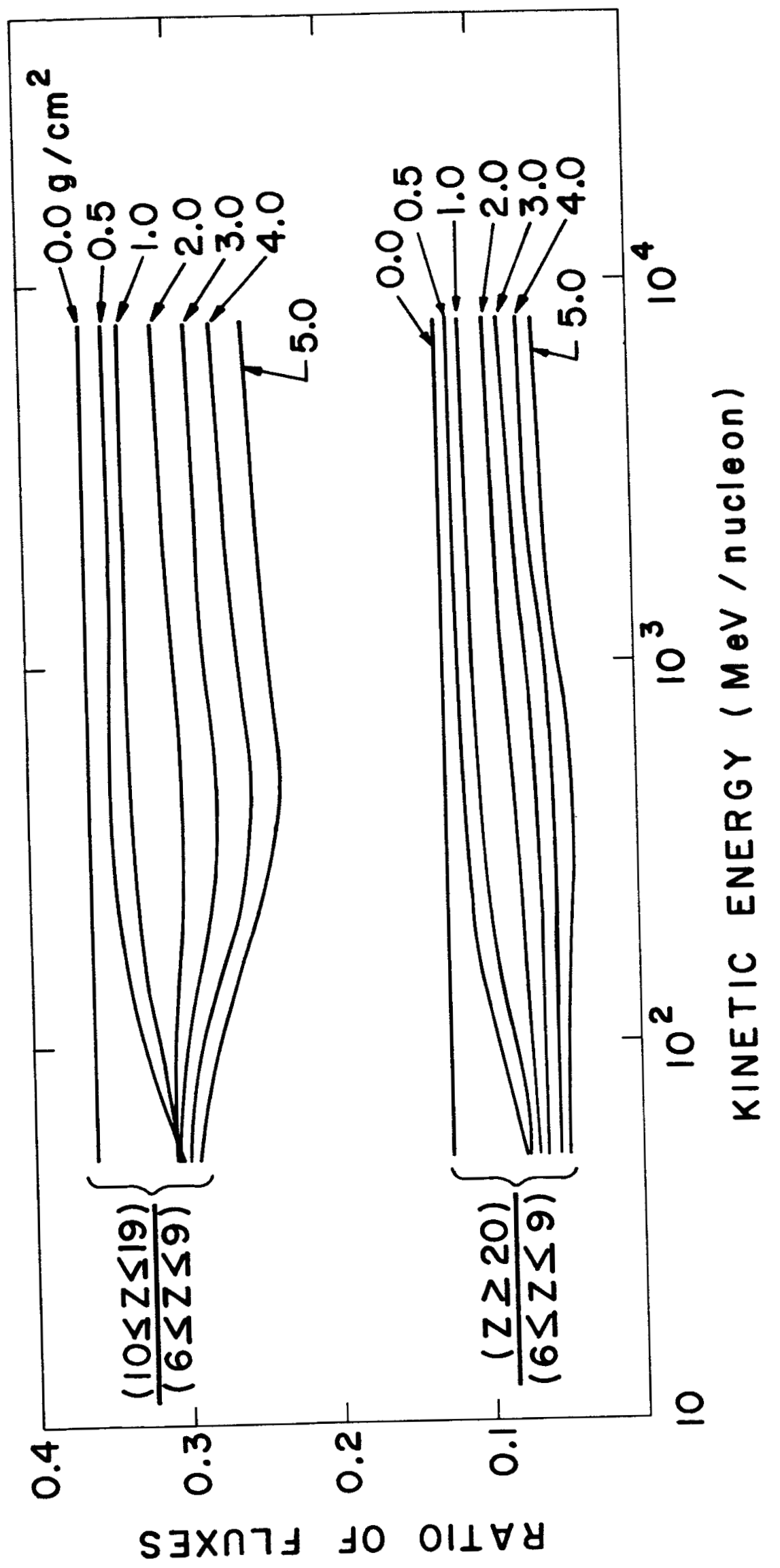




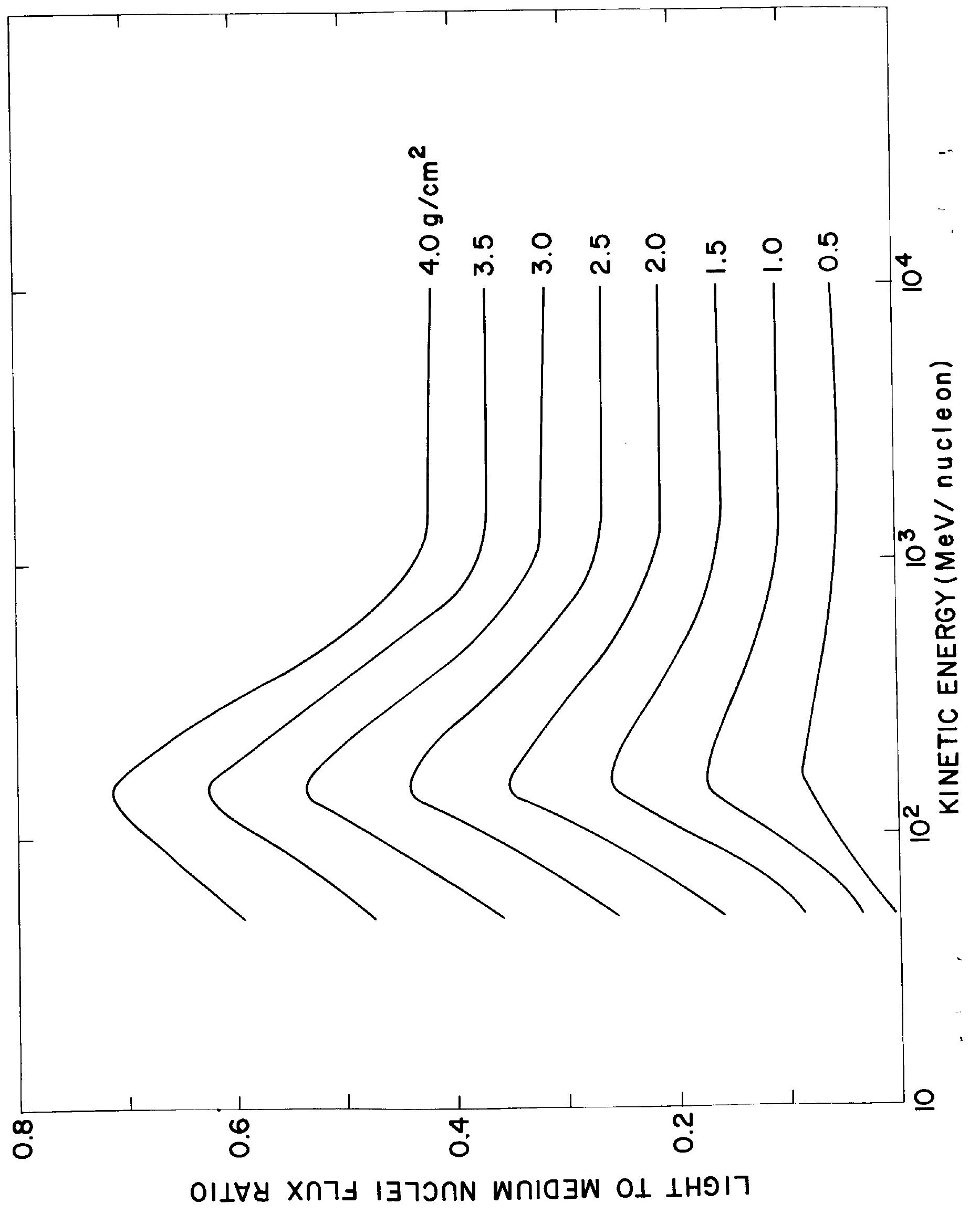




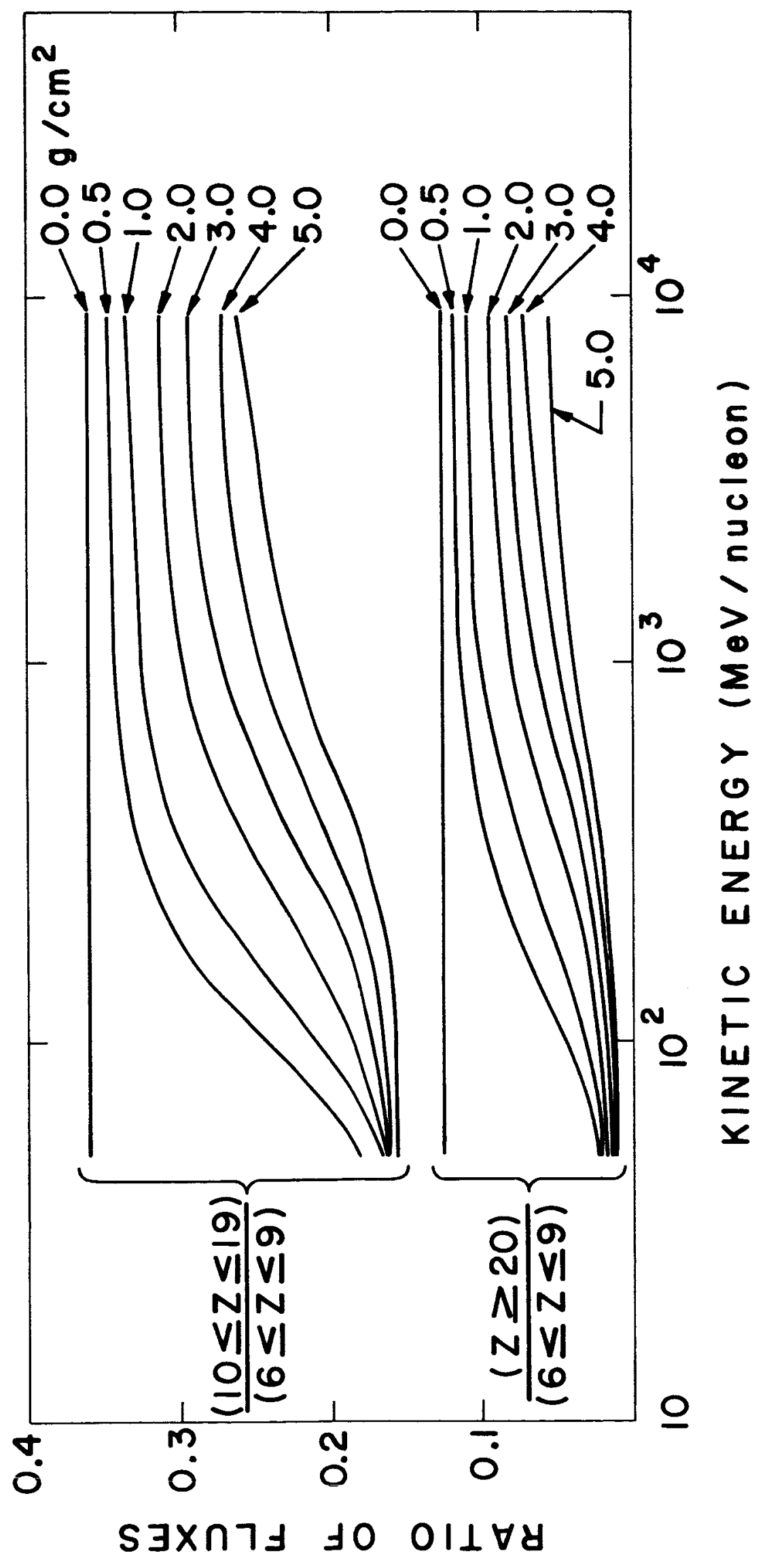




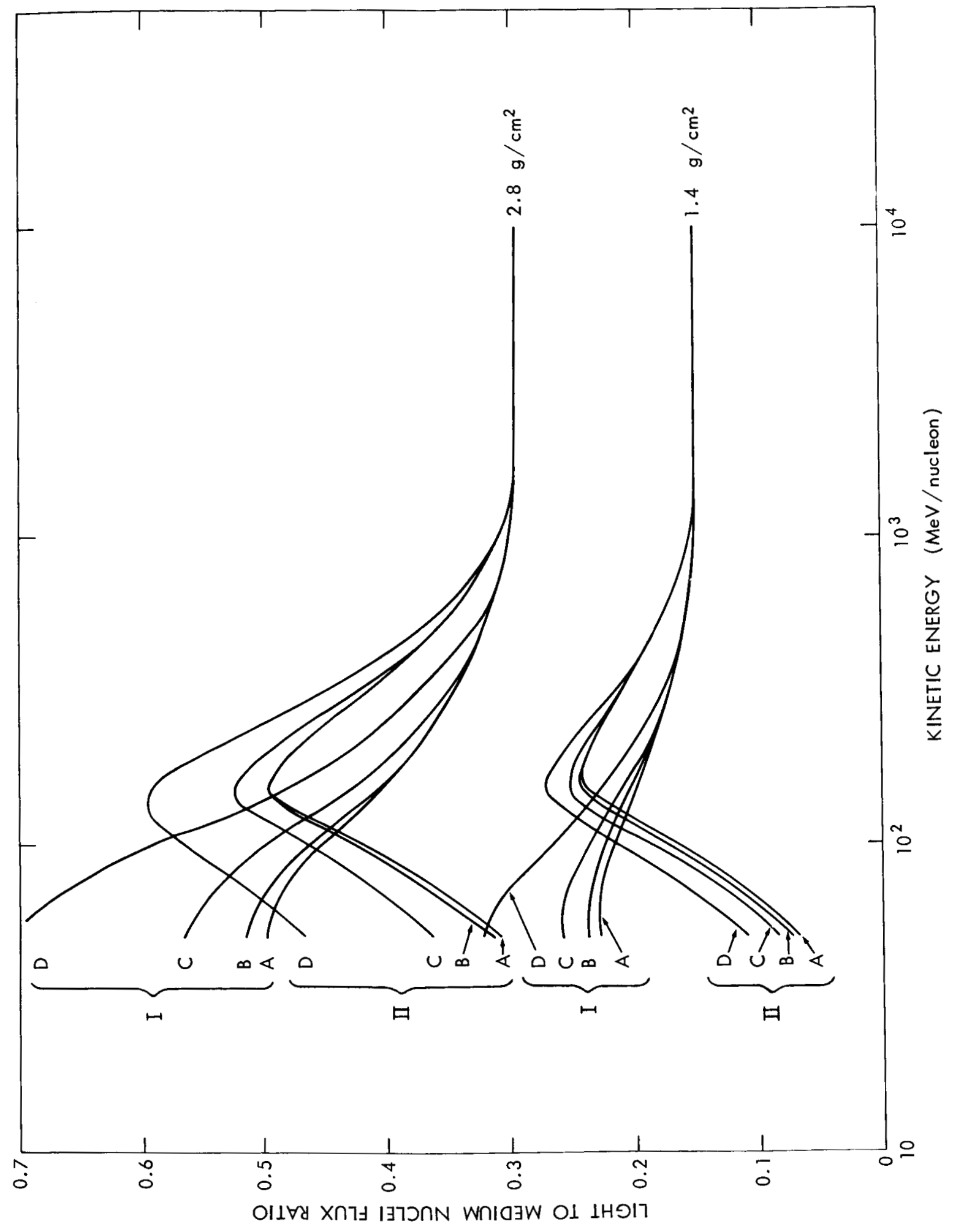




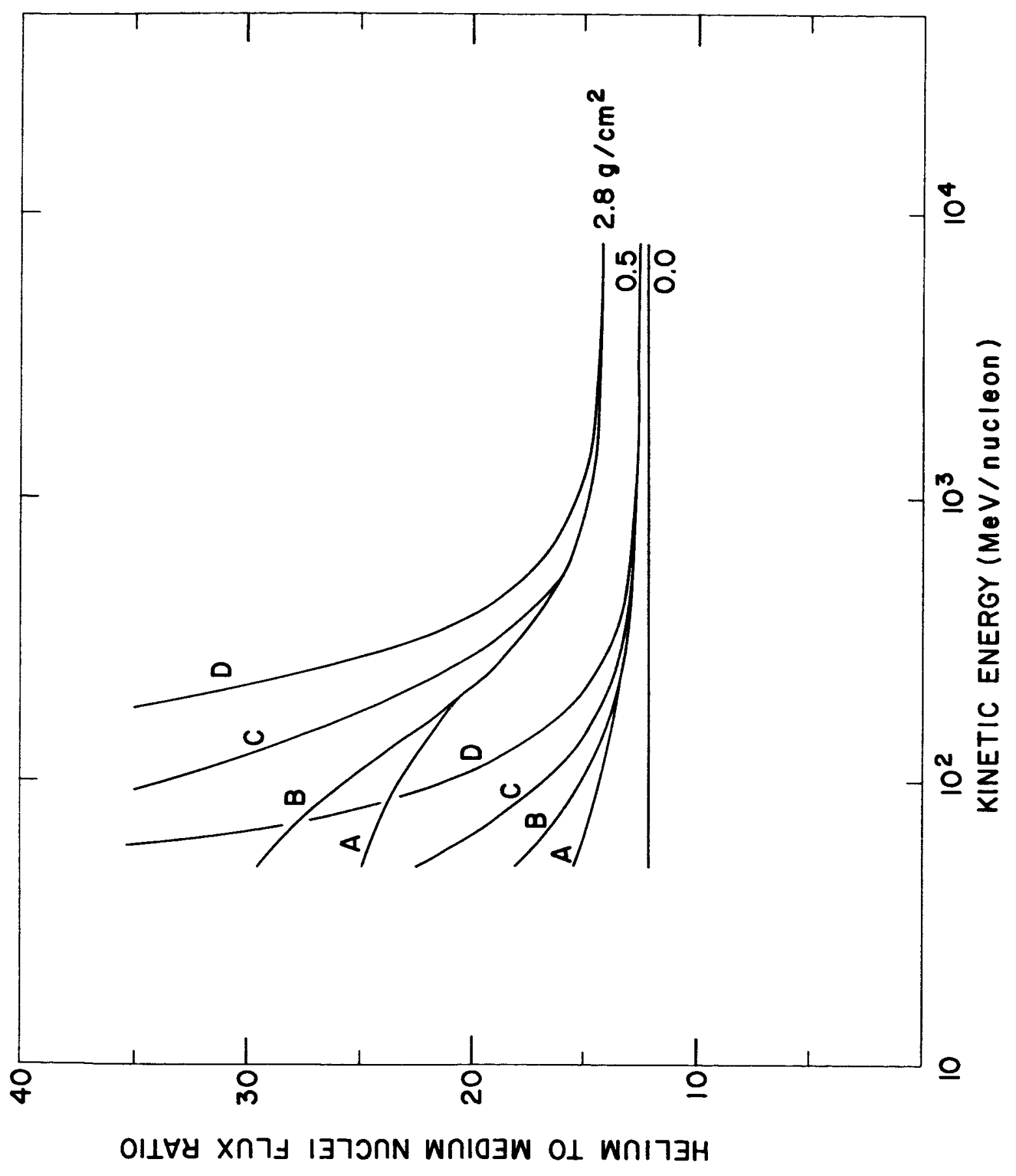




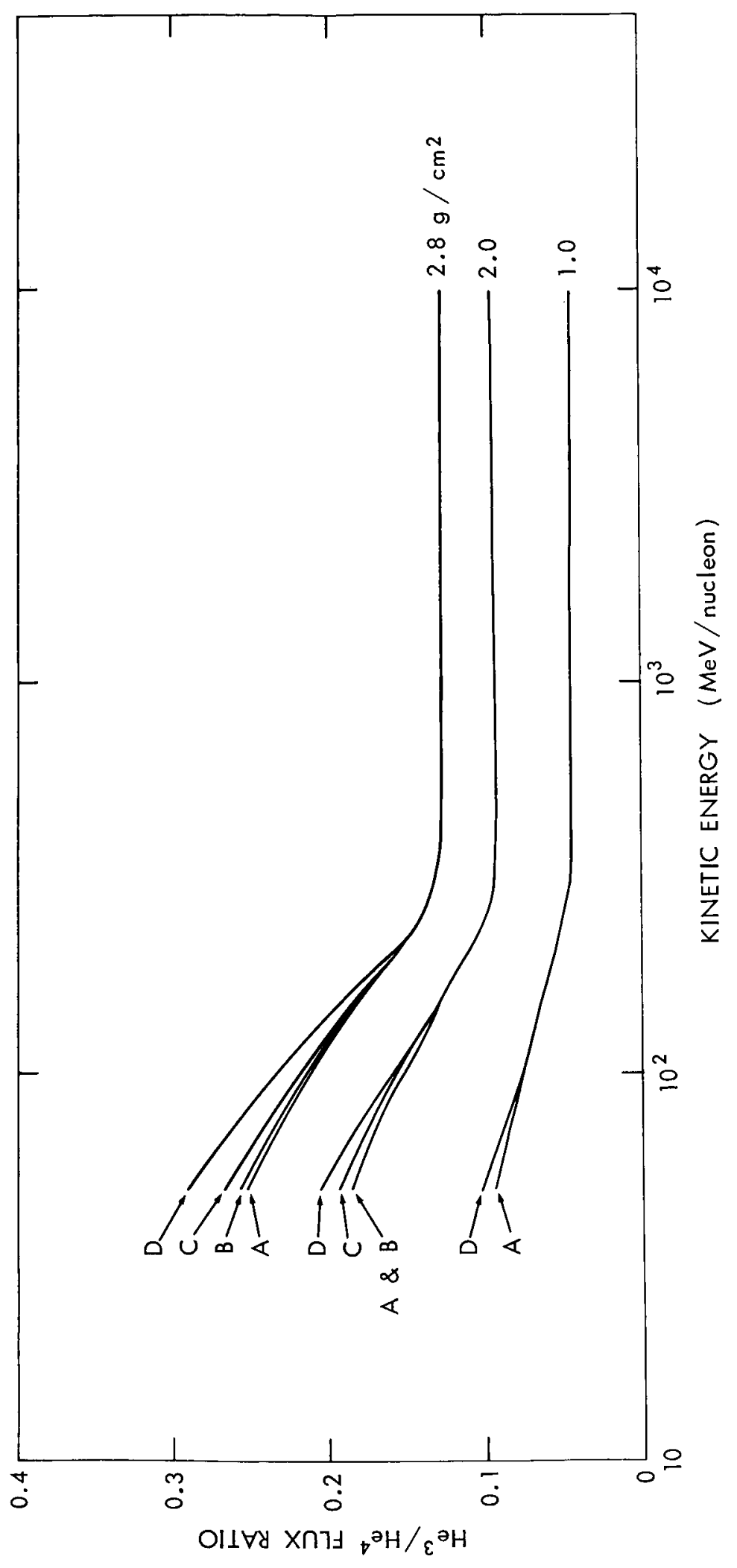




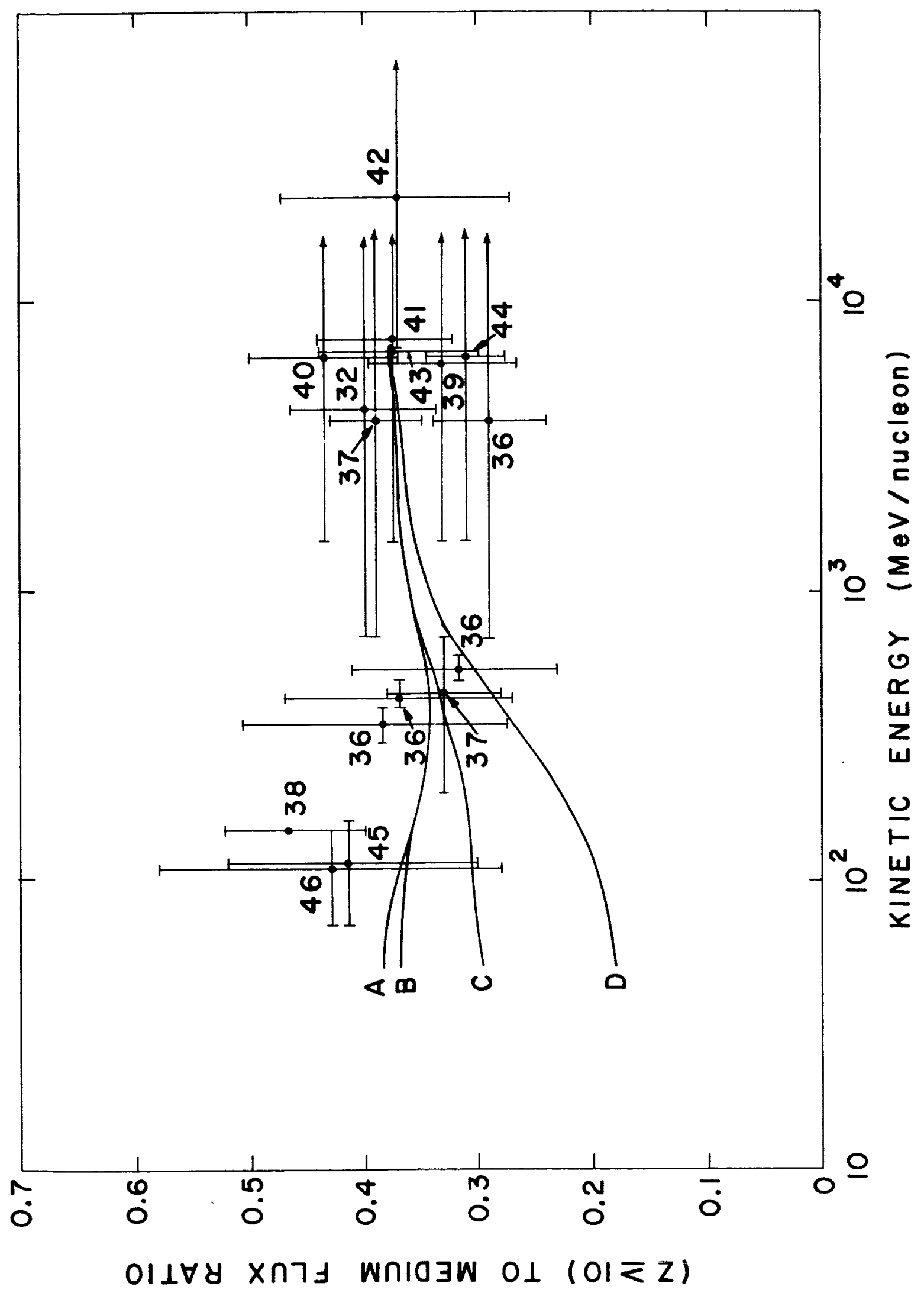




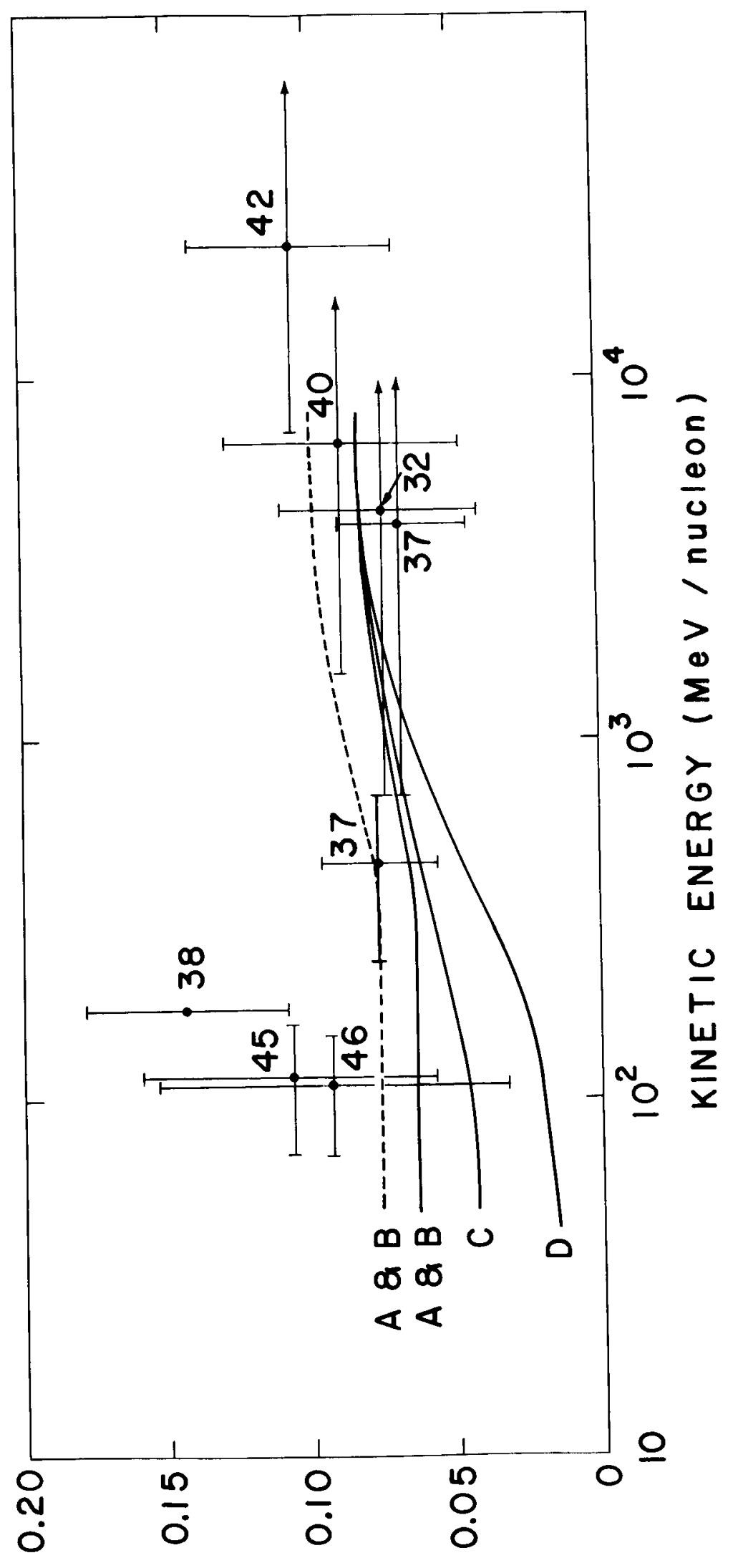

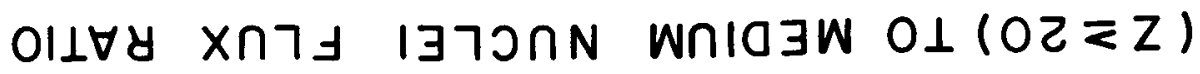


HELIUM TO MEDIUM NUCLEI FLUX RATIO

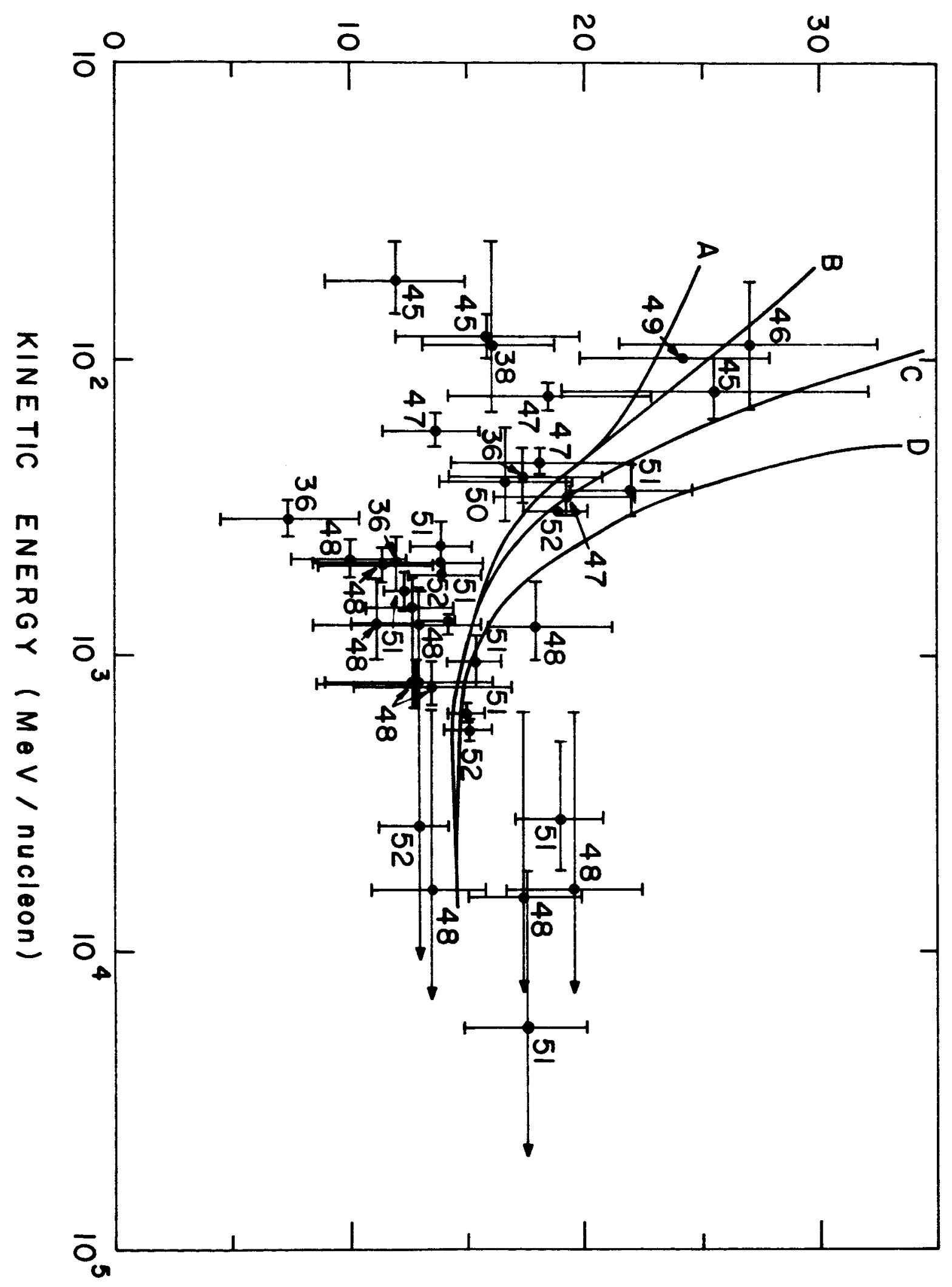




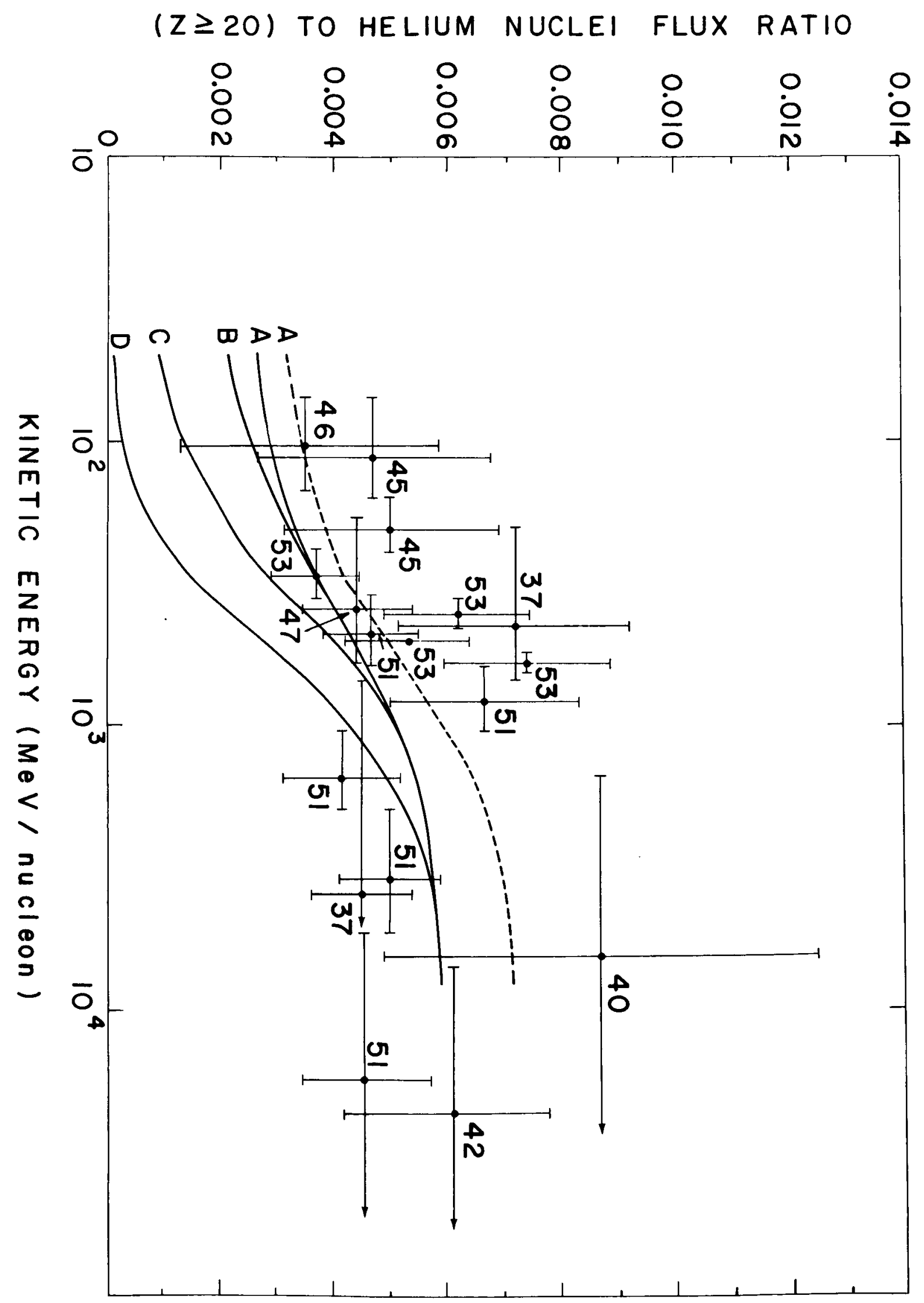




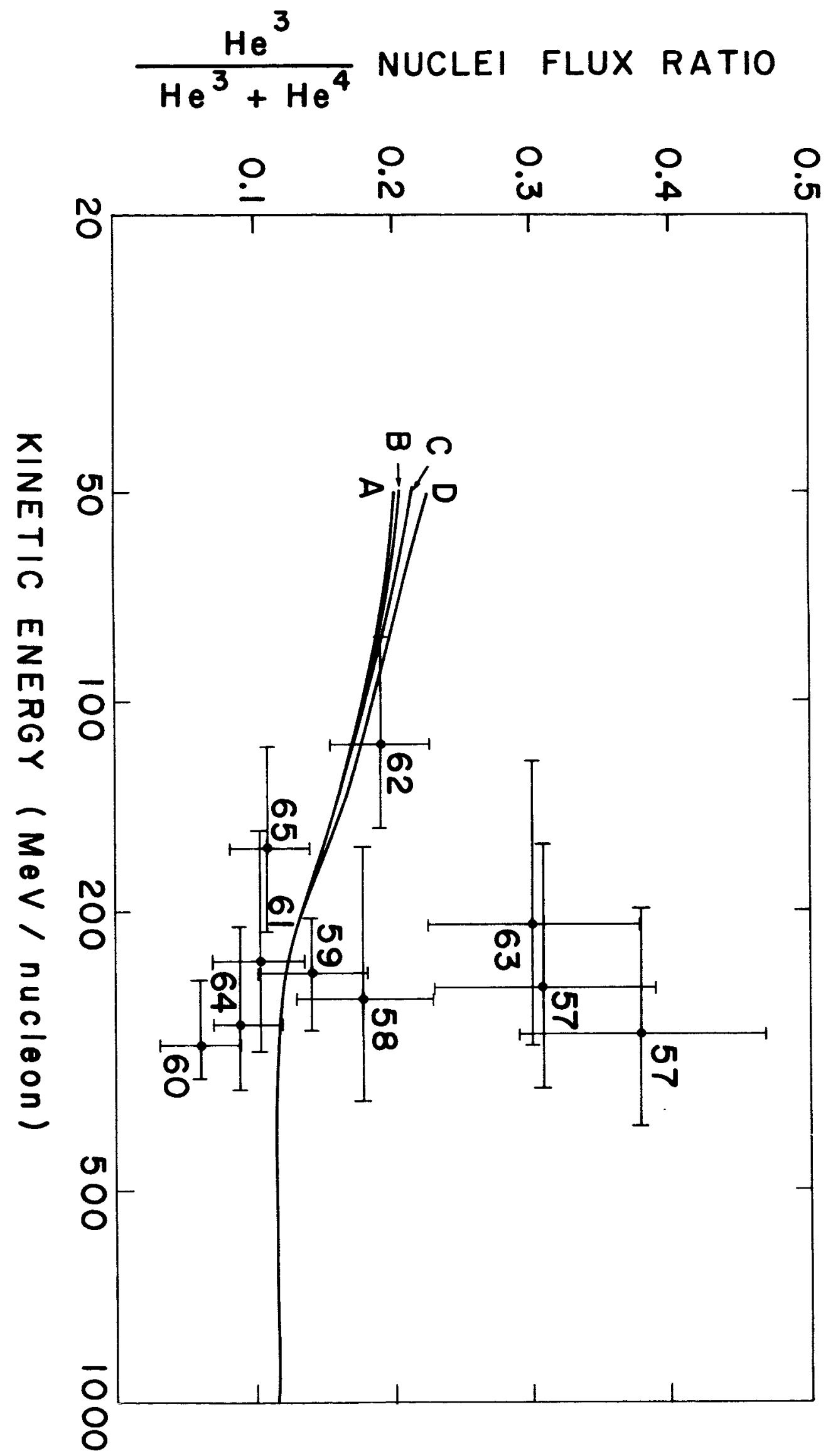




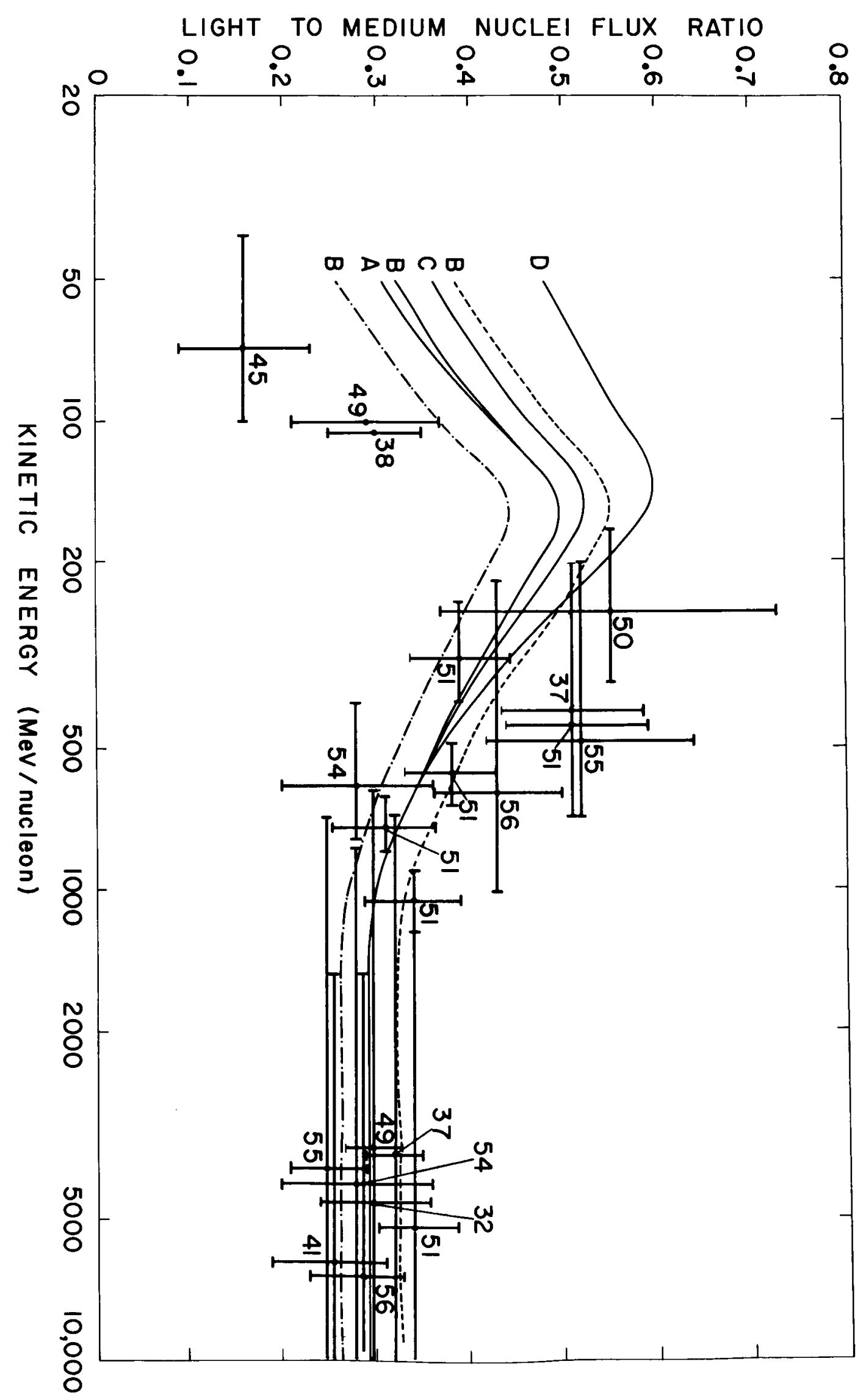

